\title{
Reshaping the tumor microenvironment with oncolytic viruses, positive regulation of the immune synapse, and blockade of the immunosuppressive oncometabolic circuitry
}

\section{Teresa Nguyen}

MD Anderson Cancer Center

Dong Ho Shin

MD Anderson Cancer Center https://orcid.org/0000-0002-0135-0817

\section{Sagar Sohoni}

MD Anderson Cancer Center

\section{Sanjay Singh}

UT MD Anderson Cancer Center

\section{Yisel Rivera-Molina}

MD Anderson Cancer Center

Hong Jiang

MD Anderson Cancer Center https://orcid.org/0000-0003-4668-5319

\section{Xuejun Fan}

MD Anderson Cancer Center

\section{Joy Gumin}

University of Texas M.D. Anderson Cancer Center

\section{Frederick Lang}

The University of Texas MD Anderson Cancer Center https://orcid.org/0000-0003-3747-873X

\section{Christopher Alvarez-Breckenridge}

MD Anderson Cancer Center

\section{Filipa Godoy-Vitorino}

University of Puerto Rico

\section{Lijie Zhai}

University Feinberg School of Medicine

\section{Erik Ladomersky}

University Feinberg School of Medicine

\section{Kristen Lauing}

University Feinberg School of Medicine

\section{Marta Alonso}


Department of Pediatrics, Clínica Universidad de Navarra, Pamplona, Spain https://orcid.org/00000002-7520-7351

\section{Derek Wainwright}

https://orcid.org/0000-0001-7232-4264

Candelaria Gomez-Manzano ( $\square$ cmanzano@mdanderson.org )

MD Anderson Cancer Center

Juan Fueyo

MD Anderson Cancer Center

\section{Article}

Keywords: Delta-24-RGDOX, tumor microenvironment, virotherapy

Posted Date: December 1st, 2021

DOI: https://doi.org/10.21203/rs.3.rs-1079989/v1

License: (c) (1) This work is licensed under a Creative Commons Attribution 4.0 International License. Read Full License 


\section{Reshaping the tumor microenvironment with oncolytic viruses, positive regulation of the immune synapse, and blockade of the immunosuppressive oncometabolic circuitry}

Teresa T. Nguyen ${ }^{1,2 \uparrow}$, Dong Ho Shin ${ }^{1,2}$, Sagar Sohoni ${ }^{1}$, Sanjay K. Singh ${ }^{3}$, Yisel RiveraMolina $^{1, \dagger \dagger}$, Hong Jiang ${ }^{1}$, Xuejun Fan ${ }^{1}$, Joy Gumin ${ }^{3}$, Frederick F. Lang ${ }^{3}$, Christopher AlvarezBreckenridge $^{3}$, Filipa Godoy-Vitorino ${ }^{4}$, Lijie Zhai ${ }^{5}$, Erik Ladomersky ${ }^{5}$, Kristen L. Lauing ${ }^{5}$, Marta M. Alonso ${ }^{6}$, Derek A. Wainwright ${ }^{5,7,8}$, Candelaria Gomez-Manzano ${ }^{1,2^{*}}$, and Juan FueyoMargareto ${ }^{1,2^{*}}$

Affiliations:

${ }^{1}$ Department of Neuro-Oncology, The University of Texas MD Anderson Cancer Center, Houston, TX, USA.

${ }^{2} \mathrm{MD}$ Anderson Cancer Center UTHealth Graduate School of Biomedical Sciences, Houston, TX, USA.

${ }^{3}$ Department of Neurosurgery, The University of Texas MD Anderson Cancer Center, Houston, TX, USA.

${ }^{4}$ Department of Microbiology and Medical Zoology, University of Puerto Rico, School of Medicine, PR, USA

${ }^{5}$ Department of Neurological Surgery, Northwestern University Feinberg School of Medicine, Chicago, IL, USA.

${ }^{6}$ Department of Pediatrics, Clinica Universidad de Navarra, Pamplona, Spain.

${ }^{7}$ Department of Medicine-Hematology/Oncology, Northwestern University Feinberg School of Medicine, Chicago, IL, USA.

${ }^{8}$ Department of Microbiology-Immunology, Northwestern University Feinberg School of Medicine, Chicago, IL, USA.

*Corresponding authors. Emails: jfueyo@ mdanderson.org; cmanzano@mdanderson.org

Current affiliation: $\dagger$ Department of Craniofacial Biology, University of Colorado Anschutz 


\section{Abstract}

35 Immune-related therapies have revolutionized the management of cancer. Oncolytic viruses are now considered part of the immunotherapy armamentarium and have shown promise in clinical

37 trials of patients with glioblastoma. These studies have suggested that tumor microenvironment

38 remodeling is required to achieve an effective response in solid tumors. Here, we showed that

39 Delta-24-RGDOX (DNX-2440), an oncolytic adenovirus expressing the T cell activator OX40L,

40 triggered antitumor immune responses. However, Delta-24-RGDOX also elicited paradoxical

41 activation of the cytokine-driven immunosuppressive IDO-kynurenine-AhR circuitry. The IDO-

42 kynurenine-AhR cascade had the dual effects of preventing optimum viral replication and

43 decreasing the virus-initiated antitumor immune response. To enhance virotherapy, we combined

44 Delta-24-RGDOX with clinically relevant IDO inhibitors. This combination therapy increased

45 the frequency of activated $\mathrm{CD}^{+} \mathrm{T}$ cells and decreased the frequencies of immunosuppressive

46 MDSC and Treg populations in animal models of gliomas and melanoma. Functional studies

47 demonstrated that the IDO blockade-dependent activation of immune cells against tumor

48 antigens could be reversed by the oncometabolite kynurenine. The concurrent targeting of

49 effectors and suppressors in the tumor immune landscape significantly prolonged the survival of

50 glioma- and melanoma-bearing mice. Our data identified the striking role of immunosuppressive

51 pathways in the resistance of solid tumors to oncolytic virotherapy. Specifically, the activity of

52 the tumor microenvironment IDO circuitry was responsible, at least partially, for the remodeling

53 of local immunosuppression after tumor infection. Combining molecular and immune-related

54 therapies may improve outcomes in human gliomas and other cancers treated with virotherapy. 


\section{Introduction}

Natural human viral infections can induce remission in several types of cancers. During the pandemic, cancer patients infected with severe acute respiratory syndrome coronavirus 2 (SARS-CoV-2) have experienced complete remissions ${ }^{1,2}$, illustrating the antitumor capacity of viruses. In one phase I study, the oncolytic virus Delta-24-RGD (DNX-2401) induced complete tumor regression in $20 \%$ of patients with recurrent glioblastoma ${ }^{3}$. Other findings in this clinical trial, including radiological signs of inflammation, pseudoprogression, and tumor infiltration by T-bet ${ }^{+} \mathrm{CD}^{+} \mathrm{T}$ cells, strongly suggested that the antitumor effects of Delta-24-RGD were due in part to an antitumor immune response. These observations agreed with preclinical studies showing that Delta-24-RGD infection induces autophagy and immunogenic cell death in glioblastoma ${ }^{4,5}$. To enhance the immune arm of this oncolytic virotherapy, we generated Delta24-RGDOX (DNX-2440), a third-generation adenovirus that includes the $\mathrm{T}$ cell activator OX40ligand (OX40L) ${ }^{6,7}$ in the backbone of Delta-24-RGD. Preclinical studies have shown that Delta24-RGDOX induces a stronger T cell-mediated antitumor effect than parental Delta-24-RGD in glioblastoma and metastatic melanoma murine models ${ }^{6,7}$. Based on these results, Delta-24RGDOX is being tested in clinical trials for the treatment of malignant gliomas (NCT03714334) and liver metastases (NCT04714983).

Infection of tumors with viruses is an efficient method of recruiting cytotoxic $\mathrm{CD} 8^{+} \mathrm{T}$ cells ${ }^{3,6,7}$; however, suppressive mechanisms within the tumor microenvironment restrain the effector immune response ${ }^{8}$. Therefore, improving the overall potency of virotherapy may require targeting inherent tumor immunosuppression while augmenting $T$ cell activation. Notably, viral infections elicit immunosuppressive mechanisms that further hinder virus-induced antitumor responses ${ }^{9}$.

Indoleamine 2,3-dioxygenase (IDO) is expressed in the tumor microenvironment and

81 induces immune privilege, an effect that is reversed by the administration of IDO inhibitors ${ }^{10}$.

82 The catabolism of tryptophan ( $\mathrm{Trp}$ ) by IDO produces kynurenine (Kyn), a direct activator of aryl

83 hydrocarbon receptor (AhR), promoting robust immunosuppression ${ }^{11,12}$. The IDO-Kyn-AhR

84 multicellular circuitry plays a fundamental role at the center of the immune synapse and acts as a 85 bridge among the main immune cell populations in tumors ${ }^{13}$. 
Activation of the IDO cascade is a major characteristic of several tumors ${ }^{14-16}$, including gliomas ${ }^{17}$, and correlates with poor prognoses ${ }^{17,18}$. In addition to its tumor role, IDO is activated in various tissues and cell subsets following cytokine stimulation during infection ${ }^{19}$.

Combinations of therapeutic strategies are probably required to overcome treatment resistance and improve the efficacy of immunotherapies in solid tumors. The main objective of

91 this work was to determine the therapeutic efficacy and immune effects of combined treatment

92 with Delta-24-RGDOX and IDO inhibitors in solid tumors. We showed that simultaneous

93 activation of the effector arm of the immune system, using oncolytic viruses and OX40-mediated

94 T cell activation, and inhibition of the suppressor arm, with IDO inhibitors, produced robust

95 remodeling of the tumor microenvironment, significantly enhancing viroimmunotherapy and

96 leading to tumor eradication in immunocompetent animal models.

\section{Results}

Delta-24-RGDOX infection results in robust transcriptional modulation of the tumor microenvironment.

Solid tumors consist of both tumor-intrinsic and tumor-extrinsic phenotypic features,

104 unbiased cellular and molecular profiling of complex tissues to dissect intratumoral

105 heterogeneity. Leveraging this approach, we performed bulk RNA-seq analyses of Delta-24-

106 RGDOX-infected murine gliomas to comprehensively examine changes in the tumor

107 transcriptome. These data showed drastic reshaping of the tumor microenvironment following

108 intratumoral infection (Fig. 1A-B). Specifically, ingenuity pathway analysis (IPA) of Delta-24-

109 RGDOX-infected tumors showed an enhancement of inflammation-related canonical pathways

110 in these tumors compared with control tumors (Fig. 1C). We also performed IPA to identify

111 altered upstream regulators in tumor treated with Delta-24-RGDOX infection. This analysis

112 identified IFN $\gamma$, TNF, ILB, IFN $\alpha$ and STAT1 as candidate regulators of the transcriptional

113 response to Delta-24-RGDOX infection (Fig. 1D). To explore whether these transcriptional

114 programs were caused by oncolytic adenovirus infection or transgenic expression of OX40L, we

115 performed similar RNA-seq analysis following infection with the parental oncolytic viral vector,

116 Delta-24-RGD. We observed a comparable pattern in tumors treated with the parental Delta-24-

117 RGD virus (Supplementary Fig. 1A-D). Crucially, these data confirmed our clinical and 
118 preclinical results, which showed tumor-infiltrating population characteristics that we observed

119 in surgical specimens from gliomas infected with Delta-24-RGD ${ }^{3}$. Similarly, our data were in

120 agreement with changes in the immune populations observed in murine models of glioma and

121 melanoma upon infection with oncolytic adenoviruses ${ }^{6,7}$. Therefore, the RNA-seq analyses were

122 consistent with previous data for human and murine tumors infected with an adenovirus obtained

123 using other methodologies.

124 Based on the rationale that IDO is triggered by IFN $\gamma^{20}$ and upregulated during viral

125 infections $^{9}$, we asked whether Delta-24-RGDOX treatment induces upregulation of the IDO

126 network. We observed that a great number of IDO-related transcripts were upregulated after

127 viroimmunotherapy, including those playing a pivotal role in maintaining glioma

128 immunosuppression such as TGF $\beta$, CTLA-4, PD-1, and GATA-3 (Fig. 1E). With respect to

129 estimated nontumor cell type abundances inferred from bulk tissue transcriptomes, the main

130 impact was a remarkable increase in the $\mathrm{CD}^{+} \mathrm{T}$ cell proportion $(P=0.0031)$ and a trend toward

131 upregulation of the natural killer (NK) cell proportion (Fig. 1F). Importantly, the use of Delta-

132 24-RGD allowed us to dissect the role of the OX40L/OX40 costimulatory axis; whereas tumors

133 infected with Delta-24-RGD showed an increase in $\mathrm{CD}^{+} \mathrm{T}$ cells, they did not show the same

134 shift in the population from $\mathrm{CD}^{+} \mathrm{T}$ cells to $\mathrm{CD}^{+} \mathrm{T}$ cells that was seen in tumors infected with

135 Delta-24-RGDOX. In addition, the increase in the NK cell population was much less prevalent in

136 tumors infected with the parental virus (Supplementary Fig. 1E). Indirectly implying that the

137 effects on these cell populations were specific, Delta-24-RGDOX infection resulted in a

138 negligible effect on the transcriptional signatures of macrophages and dendritic cells.

139 Collectively, these analyses indicated that Delta-24-RGDOX induced a robust effect on

140 the tumor microenvironment. The pleiotropic impact of Delta-24-RGDOX infection includes not

141 only the expected activation of proinflammatory pathways but also negative impacts on

142 regulators of the immune responses against viruses and tumors. Within the negative regulatory

143 network, IDO is activated by Delta-24-RGDOX infection and appears to play a critical role in

144 the re-emergence of the immunosuppressive response.

145

146 Delta-24-RGDOX elicits IDO expression and activation in gliomas.

147 We next aimed to confirm the effect of Delta-24-RGDOX on the activity of the IDO

148 pathway by assessing the production of IDO-related metabolites, such as Kyn, and the 
expression of key downstream targets of IDO, including the transcription factor $\mathrm{AhR}^{21-23}$.

150 Several proteins that are directly or indirectly activated by adenovirus infection via activation of 151 cytokines can modulate IDO expression. Of note, IDO was the first gene discovered to be 152 inducible by IFN $\gamma^{20}$, a potent mediator of antiviral immune responses. In addition, IDO is 153 regulated by other molecular mechanisms activated during infection, such as the STAT/JAK ${ }^{24}$ 154 and IFN type I transduction signaling pathways ${ }^{25-27}$.

155 In in vitro experiments, we observed a remarkable increase in IDO expression in human 156 and murine glioma cells following treatment with IFN $\gamma$ (Supplementary Fig. 2). We also 157 determined that infection of GL261-5 and GSC-005 murine glioma cells with Delta-24-RGDOX 158 triggered a six- to seven-fold increase in IDO levels, as assessed by qRT-PCR (Fig. 2A). 159 Additionally, infection of intracranial GL261-5-derived gliomas with Delta-24-RGDOX led to a 160 significant increase in IDO levels in vivo, and we observed a similar pattern in orthotopically 161 implanted breast tumors (Fig. 2B-C).

162 Kyn is a product of the metabolic activity of IDO $^{28}$, and therefore, we next sought to 163 ascertain whether infection of murine tumors with Delta-24-RGDOX increased the production of 164 this oncogenic metabolite in vivo. In agreement with the increased IDO mRNA expression 165 observed in murine glioma cell lines upon viral infection, both GL261-5- and GSC-005-derived 166 tumors infected with Delta-24-RGDOX showed a significantly higher Kyn/Trp ratio than control mouse brain tumors (Fig. 2D), as determined by liquid chromatography-mass spectrometry, 168 indicating the presence of an active IDO cascade in these tumors. The IDO-mediated catabolism of Trp and subsequent production of Kyn suggested the 170 potential activation of AhR, a key downstream target of IDO directly activated by Kyn ${ }^{11,29}$. 171 Importantly, AhR has dual functions as a transcriptional target for immune escape by viruses ${ }^{30,31}$ 172 and a promoter of immunosuppression in solid tumors, including gliomas ${ }^{32-35}$. Using AhR 173 reporter cells expressing a luciferase reporter functionally linked to an AhR-responsive promoter, 174 we assessed the transcriptional activity of AhR in a panel of glioma cell lines during active 175 adenoviral infection. We found that Delta-24-RGDOX treatment significantly increased AhR 176 transcriptional activity (Fig. 2E). Interestingly, viral infection enhanced AhR transcriptional 177 function with more efficiency in glioma cells than in control HeLa cells. Because the binding of 178 Kyn to AhR promotes AhR translocation to the nucleus ${ }^{32}$, we analyzed the expression and 179 subcellular localization of AhR in cancer cells infected with Delta-24-RGDOX using 
180 immunofluorescence. Compared with uninfected cells, infected cells showed significant

181 increases in the whole-cell and nuclear intensity of AhR as well as a remarkable increase in the

182 percentage of cells displaying nuclear AhR (Fig. 2F-H). Importantly, the Delta-24-RGDOX-

183 mediated changes were similar to the subcellular trafficking of AhR observed when uninfected

184 cultures were treated with Kyn (Fig. 2F-H).

185 These results suggested that following Delta-24-RGDOX infection, there was substantial 186 activation of the immunosuppressive IDO-Kyn-AhR cascade. The increased expression of the 187 oncometabolite Kyn and upregulation of the transcriptional activity of AhR strongly suggested

188 that infection of a tumor is followed by activation of the IDO circuitry with subsequent

189 maintenance of an immunosuppressive environment.

The Delta-24-RGDOX replication capability and cytotoxic effect are preserved under IDO inhibition.

To ascertain the effect of IDO activity specifically on virus oncolytic activity, we assessed the viral infectivity, viral replication, and virus-induced cytopathic effect of Delta-24RGDOX in a panel of human and murine glioma cell lines (Fig. 3). We observed that treating

196 glioma cultures with a direct IDO enzyme inhibitor did not modify the activity of Delta-24-

197 RGDOX in any of the human or murine cancer cell lines tested. Thus, the quantitative levels of 198 progeny virion production and expression of viral proteins were unchanged during IDO 199 inhibition (Fig. 3A, B, F, G). Corroborating these results, a timepoint analysis of cell viability 200 showed that the oncolytic effect was similar in cells treated with Delta-24-RGDOX alone or in 201 combination with IDO inhibitors (Fig. 3C, D, E, H, I). These results suggested that the potency 202 of Delta-24-RGDOX remained unchanged under conditions of IDO inhibition.

The combination of Delta-24-RGDOX and IDO inhibitors enhances immune activation in murine models of glioma.

To determine the phenotypic changes within the tumor microenvironment following the combined administration of Delta-24-RGDOX and an IDO inhibitor, we performed RNA-seq analysis of GL261-5 tumors from mice treated with PBS, an IDO inhibitor, Delta-24-RGDOX, or a combination of the IDO inhibitor and Delta-24-RGDOX. Hierarchical clustering of genes and samples showed that the transcriptome signatures of PBS- and IDO inhibitor-treated mice 
211 were not different. In contrast, Delta-24-RGDOX infection resulted in significant alterations in 212 gene pathways, making Delta-24-RGDOX-treated mice distinct from PBS- and IDO inhibitor-

213 treated mice (Fig. 4A and C). There was also a clear difference in the global transcriptome

214 signature between Delta-24-RGDOX- and combination therapy-treated mice (Fig. 4A and C).

215 Furthermore, network analyses of differentially expressed genes demonstrated that the addition

216 of IDO inhibition to Delta-24-RGDOX infection reverted the effects of the adenovirus on several

217 key immune-related transcripts that were induced during adenovirus infection, such as immune

218 checkpoint regulators, inflammation-related cytokines, Toll-like receptors 7 and 9 and

219 components of the STING pathway (Fig. 4C).

220 The predicted immune cell composition and transcriptome signature showed minimal

221 differences between PBS- and IDO inhibitor-treated brain tumors (Fig. 4B). Interestingly, upon

222 treatment with Delta-24-RGDOX, the estimated percentage of CD8 ${ }^{+} \mathrm{T}$ cells almost doubled

223 compared to that in the group treated with IDO inhibition alone (Delta-24-RGDOX: 23.7\%; IDO

224 inhibitor: 12.0\%); the combination of Delta-24-RGDOX and the IDO inhibitor (combination:

225 26.2\%; PBS: 16.3\%) did not show significant changes in the CD8+ T cell transcriptome

226 frequency compared to Delta-24-RGDOX alone (Fig. 4B). The combination therapy-treated

227 group displayed the lowest predicted frequencies of NK cells (combination: 1.9\%; IDO inhibitor:

$2283.7 \%$; RGDOX: 6.7\%), which have been shown to have antiviral activity ${ }^{36}$, and dendritic cells

229 (combination: 3.4\%; PBS: 7.4\%; IDO inhibitor: 11.4\%; RGDOX: 17.3\%), which have been

230 implicated in tumor tolerance ${ }^{37}$ (Fig. 4B).

231 The altered immune responses of combination therapy-treated mice versus Delta-24-

232 RGDOX-treated mice confirmed and complemented the results that showed increased activation

233 of the IDO network in Delta-24-RGDOX-treated mice and decreased IDO activation in

234 combination therapy-treated mice, compared to mice in the control group (Fig. 4C-D). These

235 data also demonstrated the ability of the IDO inhibitor to decrease the number of IDO-related

236 transcripts induced in tumors infected with the adenovirus (Fig. 4D). Remarkably, the

237 combination treatment decreased the expression of more than $90 \%$ of the upstream and

238 downstream IDO-related transcripts induced upon Delta-24-RGDOX treatment.

239 Together, these results indicate that the combination of IDO inhibition with Delta-24-

240 RGDOX infection modulates the tumor microenvironment by two means, promoting an adaptive

241 immune response while decreasing immunosuppression caused by virus-induced IDO activation. 
242 These collective findings strengthen our rationale for combining Delta-24-RGDOX infection

243 with IDO inhibition for the treatment of gliomas.

Combination of Delta-24-RGDOX infection with IDO inhibition prolongs the survival of glioma-bearing mice.

The results from several clinical studies using oncolytic viruses ${ }^{3,38-41}$, chimeric antigen receptor T cells ${ }^{42-44}$, or anti-PD-1 and anti-PD-L1 antibodies ${ }^{45,46}$ have shown that single cancer immunotherapies are unlikely to circumvent the immune evasion mechanisms of gliomas. Therefore, we aimed to test a three-pronged therapy in immunocompetent animal models. First, to ascertain whether IDO inhibition impairs oncolytic ability in vivo, we treated GL261-5 tumor-

252 bearing IDO-knockout (KO) mice with Delta-24-RGDOX. In this context of IDO inhibition in 253 the tumor microenvironment, Delta-24-RGDOX prolonged median survival and produced $20 \%$ 254 long-term survivors (Supplementary Fig. 3). We then compared the survival of glioma-bearing IDO-KO mice and wild-type mice treated with Delta-24-RGDOX or PBS (Fig. 5A-B). As expected, all the PBS-treated mice succumbed to their tumors, with a median survival time of 44 days, regardless of the mouse genotype. Importantly, Delta-24-RGDOX treatment resulted in a longer median survival duration and a higher percentage of long-term survivors in the IDO-KO mice than in the wild-type mice $(P=0.0142)$. To further determine the therapeutic efficacy of Delta-24-RGDOX combined with IDO inhibition, we tested this treatment in the intracranial GL261-5 tumor-bearing mouse model (Fig. 5C-D). The median survival times of the controltreated mice and IDO inhibitor-treated mice were 38.5 and 37.5 days, respectively. As expected, the mice treated with Delta-24-RGDOX had significantly prolonged survival, with a median survival time of 46.5 days. In addition, $20 \%$ of the mice treated with Delta-24-RGDOX survived for 120 days (the last timepoint tested). Notably, mice treated with a combination of Delta-24RGDOX and an IDO inhibitor had the greatest therapeutic benefit, with a median survival time of 108.5 days and a remarkable long-term survival (120 days) rate of 50\% ( $P=0.0295$; vs. Delta-24-RGDOX).

Next, we asked whether the antiglioma effect of the combined Delta-24-RGDOX and IDO inhibitor therapy is mediated by the immune system. To address this question, we

271 performed rechallenge experiments with long-term survivors in the combination therapy group 
273 As expected, treatment-naïve mice injected with GL261-5 cells died within 50 days, but the mice 274 treated with Delta-24-RGDOX and the IDO inhibitor survived 100 days with no symptoms of 275 disease. These data unequivocally indicated the immune nature of the treatment response, as the 276 combination of Delta-24-RGDOX and IDO inhibitors generated immune memory against glioma 277 antigens.

To confirm these results, we tested the combination therapy in another murine glioma model. To this end, we intracranially implanted GSC-005 cells in C57BL/6 mice and treated the 280 mice with either PBS or Delta-24-RGDOX with or without the IDO inhibitor (Fig. 5C and F).

281 The PBS- and IDO inhibitor-treated mice had median survival times of 38.5 or 42 days, 282 respectively, and $100 \%$ of the mice died due to tumor growth. As expected, treatment of these 283 brain tumor-bearing mice with Delta-24-RGDOX prolonged survival compared to control treatment, with a median survival time of 53 days $(P=0.02)$ but no long-term survivors. Similar to the GL261-5 model, the GSC-005 tumor-bearing mice treated with the combination of Delta24-RGDOX and the IDO inhibitor exhibited the longest median survival (63 days) and 44\% long-term survivors, indicating a trend of higher long-term survival rate than that achieved with Delta-24-RGDOX alone.

To test whether the combination therapy was also effective in other solid tumors, we treated intracranial B16 melanoma-bearing mice with PBS or with Delta-24-RGDOX with or without an IDO inhibitor (Fig. 5G). The mice treated with Delta-24-RGDOX alone had a median

292 survival time that was twice that of the PBS-treated mice, none of which survived to 10 days 293 after tumor implantation. The mice treated with the combination therapy had significantly 294 prolonged survival and a long-term survival rate of $14 \%$. These results indicate that the combination of Delta-24-RGDOX and an IDO inhibitor is therapeutically superior to virus 296 treatment alone in animal models of glioblastoma and intracranial melanoma.

In agreement with the survival data, analysis of tissue sections of brains harvested from

298 mice injected with either GL261-5 glioma cells or GSC-005 glioma cells in the different 299 treatment groups at 15 and 24 days showed progressive tumor regression in the mice treated with 300 the combination therapy (Fig. 5H-I; Supplementary Figs. 4-5). Additionally, neither the GL261-5 301 nor GSC-005 brain tumor-bearing animals treated with the combination therapy exhibited 302 significant changes in weight over the course of treatment, indicating the tolerability of this 303 treatment (Supplementary Fig. 6). 
In summary, antitumor treatment with the combination of Delta-24-RGDOX and an IDO inhibitor is superior to treatment with either agent alone and significantly prolongs survival, generates long-term survivors and induces antiglioma immune memory in immunocompetent animal models of cancer.

The anticancer effect of combination treatment with Delta-24-RGDOX infection plus IDO inhibition is dependent upon $\mathrm{CD4}^{+} \mathbf{T}$ cell activity.

To further understand the immune-mediated mechanisms underlying the anticancer effect

313 phenotypic changes in the lymphocyte population following treatment. Mice were treated as

314 described in Fig. 6A, and flow cytometric analyses revealed a significant influx of CD $45^{+} \mathrm{CD}^{+}$

315 T cells in both GL261-5 and GSC-005 tumor-bearing mice treated with Delta-24-RGDOX

316 compared to control-treated mice (Fig. 6B). These results were confirmed with

317 immunohistochemical assessments of CD3+ cells in the two murine brain tumor models (Fig.

318 6C; Supplementary Fig. 7). Compared with mice receiving monotherapy or control treatment,

319 those receiving Delta-24-RGDOX and an IDO inhibitor had significantly higher absolute counts

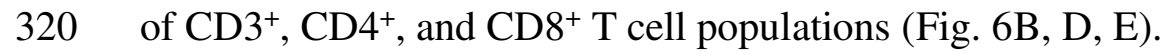

321 Based on our RNA-seq findings suggesting a link between IDO and an

322 immunosuppressive tumor microenvironment following Delta-24-RGDOX infection and on

323 previous reports of IDO activation resulting in increasing frequencies of regulatory T cells

324 (Tregs) and myeloid-derived suppressor cells (MDSCs) ${ }^{47}$, we next assessed whether these two

325 cell populations underwent changes in treated tumors. Gene set enrichment analyses of RNA-seq

326 data showed positive enrichment of gene sets associated with Tregs (normalized enrichment

327 score of $1.375, P=0.0$, FDR q-value $=0.0439$ ) and MDSCs (normalized enrichment score of

$3281.352, P=0.0$, FDR q-value $=0.0642)$ in tumors treated with Delta-24-RGDOX compared with

329 control-treated tumors (Fig. 6F, left panels). In contrast, the combination of Delta-24-RGDOX

330 infection with IDO inhibition led to a reversal of these findings, shifting the tumor

331 microenvironment away from an immunosuppressive phenotype (Fig. 6F, right). These results

332 were confirmed by flow cytometric analyses of the tumor microenvironment of virus- and

333 combination-treated mice (Fig. 6G). Importantly, whereas Delta-24-RGDOX alone increased 
334 Treg and MDSC populations, Delta-24-RGDOX plus the IDO inhibitor significantly decreased 335 these crucial immunosuppressive populations.

336 Since $\mathrm{CD}^{+}{ }^{+} \mathrm{T}$ cells constitute the target of the OX40 ligand/receptor synapse ${ }^{48}$, we aimed 337 to understand the extent to which these cells contributed to the therapeutic efficacy of the 338 combination of Delta-24-RGDOX infection and IDO inhibition in murine glioblastoma. To this 339 end, we performed a survival experiment in which CD4 ${ }^{+} \mathrm{T}$ cells were depleted using anti-CD4 340 antibodies (Fig. 7A-C). Our results showed that in mice with a decreased population of CD4 ${ }^{+} \mathrm{T}$ 341 cells, the antiglioma effect of the combination treatment was abolished, and the median survival 342 of the combination-treated $\mathrm{CD}^{+} \mathrm{T}$ cell-depleted mice was similar to that of control-treated mice 343 (Fig. 7D). This loss of the anticancer effect indicates that the combined therapy depends on the $344 \mathrm{CD}^{+} \mathrm{T}$ helper cell population to induce maximal anticancer effects and produce long-term survivors. We also examined the effect of depletion of Tregs, a CD4 ${ }^{+}$cell population, on the 346 therapeutic activity of the combination treatment. We observed that depleting $85 \%$ of the CD25 347 population (Fig. 7B-C) had no significant deleterious effect on the survival of mice receiving the combination treatment (Fig. 7D). These results were consistent with the observations that the combination treatment induced a significant reduction in Tregs (Fig. 6G) and suggested that blocking IDO already caused functionally relevant inactivation of Tregs. Additionally, these analyses also suggested that the effect observed with the depletion of CD4+ cells was most likely 352 due to the reduction in the CD4+ T helper cell population. In summary, our data indicated that the combination of Delta-24-RGDOX infection with 354 IDO inhibition resulted in intratumoral infiltration of several T cell populations, such as $355 \mathrm{CD} 45^{+} \mathrm{CD}^{+}, \mathrm{CD} 45^{+} \mathrm{CD} 3^{+} \mathrm{CD}^{+}$and $\mathrm{CD} 45^{+} \mathrm{CD}^{+} \mathrm{CD}^{+}$cells. Of interest, we detected 356 paradoxical activation of immunosuppressive populations, including MDSCs and Tregs, by 357 Delta-24-RGDOX, which was partially reversed by the addition of IDO inhibitors. Furthermore, 358 the depletion of $\mathrm{CD}^{+}$cells, the main target of the OX40 ligand/receptor synapse ${ }^{48}$, abrogated the 359 antitumor efficacy of this combination therapy. These findings underscored the critical role of 360 the $\mathrm{CD}^{+} \mathrm{T}$ cell population, but not the Treg population, in the eradication of tumors treated with 361 Delta-24-RGDOX and an IDO inhibitor. 


\section{Treatment with Delta-24-RGDOX infection and IDO inhibition led to functional activation}

365 of antitumor immune cells.

To study the function of $\mathrm{T}$ cells in response to treatment, we performed coculture-based functional immune assays. Thus, splenocytes from GL261-5 tumor-bearing mice treated with Delta-24-RGDOX with or without IDO inhibitors were cocultured with uninfected or Delta-24RGDOX-infected glioma cells to assess antitumor immune responses (Fig. 8A-D). We showed that splenocytes isolated from mice treated with the combination therapy displayed robust immune activation against Delta-24-RGDOX-infected glioma cells, as determined by measuring

372 the secreted levels of the Th1 cytokines IFN $\gamma$ and IL-2 (Fig. 8C). Importantly, we detected the

373 same trend of immune activation against uninfected glioma cells (Fig. 8B), suggesting that tumor

374 infection resulted in antigen spreading that led to recognition of tumor antigens. We also evaluated this T-cell activation at an earlier timepoint, which further confirmed the antitumor activation of splenocytes against uninfected cells (Supplementary Fig. 8).

We then sought to dissect the negative role of Kyn in $\mathrm{T}$ cell activation using similar functional assays (Fig. 8D). To this end, we challenged the effect of IDO inhibition by enriching the culture medium with Kyn and then examined the activity of splenocytes cocultured with glioma cells ${ }^{49}$. As expected, the secretion of IL-2 was increased in cocultures of splenocytes isolated from virus-treated mice compared to control-treated mice when either uninfected cells or virus-infected cells were included, validating the virus-induced activation of $\mathrm{T}$ cells against both tumor antigens and viral antigens (Fig. 8D). Interestingly, the addition of Kyn partially decreased $\mathrm{T}$ cell functionality by reducing IL-2 secretion, evidencing that Kyn had a negative effect on virusactivated splenocytes (Fig. 8D) ${ }^{49}$.

These functional assays showed the robust effect of IDO inhibition on the activation of immune cells against cancer cells and the significant reversion of this immune activation by downstream activators of the IDO signaling circuitry. In addition, these data indirectly suggested that the IDO-Kyn circuitry has a role in counteracting the antitumoral virus-mediated activation of T cells. 


\section{Discussion}

A recent plethora of clinical trials in adults and children with brain tumors have shown that oncolytic viruses prolong the survival of a small percentage of patients $(<20 \%)^{3,38-41}$. Importantly, these studies have also shown that viroimmunotherapy induces $\mathrm{T}$ cell infiltration into brain tumors ${ }^{3,38-41}$. Such findings support the paradigm-shifting concept that complete tumor debulking by virotherapy requires the elicitation of antitumor immune responses following the initial oncolytic effect. Therefore, further enhancement of the immune arm of this treatment approach may be required to increase the percentage of positive responders ${ }^{50}$. Here, we showed that the infection of a tumor with the armed oncolytic adenovirus Delta-24-RGDOX was followed by a striking activation of the immune response. Intriguingly, RNA-seq analyses further revealed that Delta-24-RGDOX infection promoted the paradoxical activation of immunosuppressive pathways, including the IDO cascade, and immunosuppressive populations, such Tregs and MDSCs. This double-edged activation of positive and negative immune regulators is occasionally observed during viral infections ${ }^{51-53}$. As exemplified by the transcriptional signature of the treated gliomas in this study, viral infection activates immune checkpoint regulators, including CTLA-4, PD-1, and IDO ${ }^{54}$. This counterintuitive effect of tumor infection was confirmed by in vitro and in vivo studies showing that Delta-24-RGDOX infection is followed by the upregulation and activation of the IDO cascade, a notable finding that is in agreement with previous reports showing that IDO is upregulated in human glioma cells upon exposure to the major antiviral cytokine IFN $\gamma$, which is also a transcriptional regulator of IDO $^{20,55}$. Thus, our results are consistent with the cell and tissue upregulation of IDO following cytokine stimulation in the context of infection, autoimmunity, and cancer ${ }^{19}$. Downstream of IDO, AhR is activated by viral infection ${ }^{56}$ and induces immunosuppression ${ }^{11,12,57-59}$, which in turn can favor cancer progression. In human patients with glioblastoma, the expression of AhR is associated with a poor prognosis ${ }^{60}$. Thus, the initial effect of Delta-24-RGDOX may be counteracted by the expression of the IDO-AhR cascade and the subsequent prevention of an antitumor immune response. Therefore, we hypothesized that targeting the IDO pathway should have a double-positive effect: on the one hand, improving virotherapy and, on the other hand, decreasing intrinsic tumor immunosuppression (Fig. 9).

Of further clinical relevance, IDO and AhR are potential druggable targets in malignant gliomas $^{17,60-62}$. In this work, we demonstrated that Delta-24-RGDOX combined with IDO 
428 inhibitors induced prolonged survival and generated a higher rate of long-term survival than

429 control treatments in murine models of glioma and intracranial melanoma. The addition of IDO

430 inhibitors is required to induce anticancer effects in several models of cancer. For example,

431 HPV16-E6-E7/HRAS-driven lung epithelial cancer was suppressed by only the combination of 432 HPV16 E7 vaccines, OX40 agonists, and IDO inhibitors ${ }^{63}$. Furthermore, Berrong et al ${ }^{63}$ showed 433 that while the addition of anti-OX40 to an antigen-specific cancer vaccine moderately enhanced 434 therapeutic efficacy, it was the addition of an IDO inhibitor to this treatment that eventually led 435 to complete regression of established tumors in $60 \%$ of treated mice. Additionally, in agreement 436 with our data, Sagiv-Barfi and colleagues combined intratumoral delivery of an adenovirus437 related TLR9 ligand with OX40 activation to increase anticancer T cell responses ${ }^{64}$. 438 The IDO-Kyn-AhR pathway potentiates systemic toxicity during viral infection ${ }^{30,31}$. 439 Moreover, activation of the IDO-Kyn-AhR cascade worsens influenza virus infection ${ }^{65-68}$. In 440 addition, constitutive AhR activation reduces the type I IFN antiviral response ${ }^{23}$. Therefore, the 441 inhibition of the IDO circuitry should likely limit the potential systemic toxicity of Delta-24442 RGDOX in clinical trials. In this study, we did not observe any sign of toxicity in mice treated 443 with the combination therapy.

444 The OX40 pathway is predominantly active in CD4 ${ }^{+} \mathrm{T}$ cells ${ }^{69}$. In our models, the 445 anticancer effect of Delta-24-RGDOX infection combined with IDO inhibition was due to the 446 elicitation of a robust antitumor immune response. Thus, we observed increased absolute counts 447 of tumor infiltrating $\mathrm{CD}^{+}$and $\mathrm{CD}^{+} \mathrm{T}$ lymphocytes in combination treated tumors. These 448 observations aligned with our functional studies showing that Kyn negatively regulated T cell 449 responses to viral or tumor antigens. Furthermore, antibody depletion of $\mathrm{CD}^{+} \mathrm{T}$ cells abolished 450 the antitumor effect of the combination therapy, but the anticancer effect was unaffected when 451 Tregs were depleted. Together, the $\mathrm{T}$ cell depletion studies indicate that the $\mathrm{T}$ helper cell 452 response is required to elicit antitumor immune responses and that IDO inhibition mimics Treg 453 inhibition.

454 Our data also indicated that the virus-mediated upregulation of the IDO cascade was 455 associated with intriguing increases in the MDSC and Treg populations, which were partially 456 counteracted by IDO inhibition. In this regard, IDO orchestrates immunosuppressive effects 457 through Treg-dependent recruitment and activation of $\operatorname{MDSCs}^{47}$. Furthermore, IDO expression in 458 solid tumors is strongly associated with Treg and MDSC infiltration, which can be reversed by 
459 IDO inhibition ${ }^{47}$. Moreover, IDO activity inhibits the proliferation of antigen-specific T 460 lymphocytes, inducing tumor tolerance ${ }^{59,70-73}$, and further inhibits $\mathrm{T}$ cell-based adaptive

461 immunity by promoting the differentiation of Tregs in tumors ${ }^{15,74-76}$, which can be induced by $462 \mathrm{Kyn}^{11}$ and result in the suppression of antigen-specific $\mathrm{T}$ cell responses ${ }^{49,77}$. It is important to 463 note that in our cell system, the addition of Kyn to cocultures of splenocytes from treated mice 464 and cancer cells efficiently suppressed the activation of the immune cells elicited by IDO 465 inhibition.

466 In conclusion, our findings reveal that immunosuppressive pathways play a prominent 467 role in the resistance of solid tumors to oncolytic virotherapy. Furthermore, the activity of the 468 tumor microenvironment IDO circuitry is responsible, at least partially, for the remodeling of 469 local immunosuppression after tumor infection. Combining molecular and immune-related 470 therapies may improve outcomes in human gliomas and other cancers treated with virotherapy. 471 These highly translatable studies should propel the development of a clinical trial to test the 472 safety and efficacy of the combination of oncolytic adenoviruses armed with $\mathrm{T}$ cell activators 473 and IDO inhibitors in patients with glioblastoma and other solid tumors.

\section{Data availability}




\section{References}

4811 Challenor, S. \& Tucker, D. SARS-CoV-2-induced remission of Hodgkin lymphoma. $\mathrm{Br} \mathrm{J}$

482

483

2 Sollini, M., Gelardi, F., Carlo-Stella, C. \& Chiti, A. Complete remission of follicular lymphoma after SARS-CoV-2 infection: from the "flare phenomenon" to the "abscopal effect". Eur J Nucl Med Mol Imaging, doi:10.1007/s00259-021-05275-6 (2021).

3 Lang, F. F. et al. Phase I Study of DNX-2401 (Delta-24-RGD) Oncolytic Adenovirus: Replication and Immunotherapeutic Effects in Recurrent Malignant Glioma. J Clin Oncol, JCO2017758219, doi:10.1200/JCO.2017.75.8219 (2018).

4 Jiang, H. \& Fueyo, J. Healing after death: antitumor immunity induced by oncolytic adenoviral therapy. Oncoimmunology 3, e947872, doi:10.4161/21624011.2014.947872 (2014).

5 Jiang, H. et al. Oncolytic adenovirus research evolution: from cell-cycle checkpoints to immune checkpoints. Current opinion in virology 13, 33-39, doi:10.1016/j.coviro.2015.03.009 (2015).

6 Jiang, H. et al. Oncolytic Adenovirus and Tumor-Targeting Immune Modulatory Therapy Improve Autologous Cancer Vaccination. Cancer Research 77, 3894-3907, doi:10.1158/0008-5472.can-17-0468 (2017).

7 Jiang, H. et al. Localized Treatment with Oncolytic Adenovirus Delta-24-RGDOX Induces Systemic Immunity against Disseminated Subcutaneous and Intracranial Melanomas. Clin Cancer Res 25, 6801-6814, doi:10.1158/1078-0432.CCR-19-0405 (2019).

8 Saleh, R. \& Elkord, E. Acquired resistance to cancer immunotherapy: Role of tumormediated immunosuppression. Semin Cancer Biol 65, 13-27, doi:10.1016/j.semcancer.2019.07.017 (2020).

9 Schmidt, S. V. \& Schultze, J. L. New Insights into IDO Biology in Bacterial and Viral Infections. Front Immunol 5, 384, doi:10.3389/fimmu.2014.00384 (2014).

10 Uyttenhove, C. et al. Evidence for a tumoral immune resistance mechanism based on tryptophan degradation by indoleamine 2,3-dioxygenase. Nat Med 9, 1269-1274, doi:10.1038/nm934 (2003).

11 Mezrich, J. D. et al. An interaction between kynurenine and the aryl hydrocarbon receptor can generate regulatory T cells. J Immunol 185, 3190-3198, doi:10.4049/jimmunol.0903670 (2010).

12 Quintana, F. J. et al. Control of T(reg) and T(H)17 cell differentiation by the aryl hydrocarbon receptor. Nature 453, 65-71, doi:10.1038/nature06880 (2008).

13 Routy, J. P., Routy, B., Graziani, G. M. \& Mehraj, V. The Kynurenine Pathway Is a Double-Edged Sword in Immune-Privileged Sites and in Cancer: Implications for Immunotherapy. Int J Tryptophan Res 9, 67-77, doi:10.4137/IJTR.S38355 (2016).

14 Munn, D. H. et al. Expression of indoleamine 2,3-dioxygenase by plasmacytoid dendritic cells in tumor-draining lymph nodes. J Clin Invest 114, 280-290, doi:10.1172/JCI21583 (2004).

15 Curti, A. et al. Modulation of tryptophan catabolism by human leukemic cells results in the conversion of CD25- into CD25+ T regulatory cells. Blood 109, 2871-2877, doi:10.1182/blood-2006-07-036863 (2007). 
52416 Mansfield, A. S. et al. Simultaneous Foxp3 and IDO expression is associated with

17 Wainwright, D. A. et al. IDO expression in brain tumors increases the recruitment of regulatory T cells and negatively impacts survival. Clin Cancer Res 18, 6110-6121, doi:10.1158/1078-0432.CCR-12-2130 (2012).

18 Du, L. et al. Both IDO1 and TDO contribute to the malignancy of gliomas via the KynAhR-AQP4 signaling pathway. Signal Transduct Target Ther 5, 10, doi:10.1038/s41392019-0103-4 (2020).

19 Jaronen, M. \& Quintana, F. J. Immunological Relevance of the Coevolution of IDO1 and AHR. Front Immunol 5, 521, doi:10.3389/fimmu.2014.00521 (2014).

20 Yoshida, R., Imanishi, J., Oku, T., Kishida, T. \& Hayaishi, O. Induction of pulmonary indoleamine 2,3-dioxygenase by interferon. Proceedings of the National Academy of Sciences of the United States of America 78, 129-132, doi:10.1073/pnas.78.1.129 (1981).

21 Platten, M., von Knebel Doeberitz, N., Oezen, I., Wick, W. \& Ochs, K. Cancer Immunotherapy by Targeting IDO1/TDO and Their Downstream Effectors. Front Immunol 5, 673, doi:10.3389/fimmu.2014.00673 (2014).

22 Litzenburger, U. M. et al. Constitutive IDO expression in human cancer is sustained by an autocrine signaling loop involving IL-6, STAT3 and the AHR. Oncotarget 5, 10381051, doi:10.18632/oncotarget.1637 (2014).

23 Yamada, T. et al. Constitutive aryl hydrocarbon receptor signaling constrains type I interferon-mediated antiviral innate defense. Nat Immunol 17, 687-694, doi:10.1038/ni.3422 (2016).

24 Arumuggam, N., Bhowmick, N. A. \& Rupasinghe, H. P. A Review: Phytochemicals Targeting JAK/STAT Signaling and IDO Expression in Cancer. Phytother Res 29, 805817, doi:10.1002/ptr.5327 (2015).

25 Boasso, A., Hardy, A. W., Anderson, S. A., Dolan, M. J. \& Shearer, G. M. HIV-induced type I interferon and tryptophan catabolism drive T cell dysfunction despite phenotypic activation. PLoS One 3, e2961, doi:10.1371/journal.pone.0002961 (2008).

26 Schroecksnadel, K. et al. Indoleamine-2, 3-dioxygenase and other interferon-gammamediated pathways in patients with human immunodeficiency virus infection. Curr Drug Metab 8, 225-236, doi:10.2174/138920007780362608 (2007).

27 Sage, L. K., Fox, J. M., Mellor, A. L., Tompkins, S. M. \& Tripp, R. A. Indoleamine 2,3dioxygenase (IDO) activity during the primary immune response to influenza infection modifies the memory T cell response to influenza challenge. Viral Immunol 27, 112-123, doi:10.1089/vim.2013.0105 (2014).

28 Zhai, L. et al. Molecular Pathways: Targeting IDO1 and Other Tryptophan Dioxygenases for Cancer Immunotherapy. Clin Cancer Res 21, 5427-5433, doi:10.1158/10780432.CCR-15-0420 (2015).

29 Murray, I. A., Patterson, A. D. \& Perdew, G. H. Aryl hydrocarbon receptor ligands in cancer: friend and foe. Nat Rev Cancer 14, 801-814, doi:10.1038/nrc3846 (2014).

30 Barroso, A. et al. The Aryl Hydrocarbon Receptor Modulates Production of Cytokines and Reactive Oxygen Species and Development of Myocarditis during Trypanosoma cruzi Infection. Infect Immun 84, 3071-3082, doi:10.1128/IAI.00575-16 (2016). 
$56831 \quad$ Sanchez, Y. et al. The unexpected role for the aryl hydrocarbon receptor on susceptibility to experimental toxoplasmosis. J Biomed Biotechnol 2010, 505694, doi:10.1155/2010/505694 (2010).

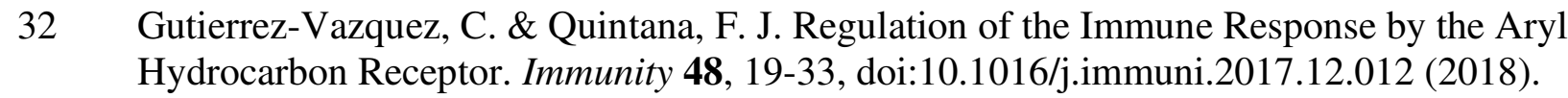

33 Rothhammer, V. \& Quintana, F. J. The aryl hydrocarbon receptor: an environmental sensor integrating immune responses in health and disease. Nature reviews. Immunology

575

576 19, 184-197, doi:10.1038/s41577-019-0125-8 (2019).

577

578

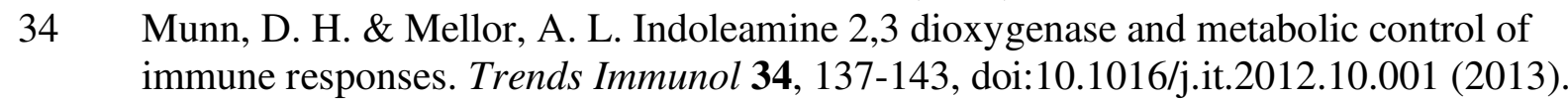

35 Prendergast, G. C., Mondal, A., Dey, S., Laury-Kleintop, L. D. \& Muller, A. J. Inflammatory Reprogramming with IDO1 Inhibitors: Turning Immunologically Unresponsive 'Cold' Tumors 'Hot'. Trends Cancer 4, 38-58, doi:10.1016/j.trecan.2017.11.005 (2018). oncolytic virotherapy. Cytokine Growth Factor Rev 56, 59-68, doi:10.1016/j.cytogfr.2020.06.005 (2020). Mediated Dendritic Cell Tolerization in Immune Evasion. Front Immunol 10, 2876, doi:10.3389/fimmu.2019.02876 (2019).

38 Friedman, G. K. et al. Oncolytic HSV-1 G207 Immunovirotherapy for Pediatric HighGrade Gliomas. N Engl J Med, doi:10.1056/NEJMoa2024947 (2021).

39 Cloughesy, T. F. et al. Phase 1 trial of vocimagene amiretrorepvec and 5-fluorocytosine for recurrent high-grade glioma. Sci Transl Med 8, 341ra375, doi:10.1126/scitranslmed.aad9784 (2016).

40 Desjardins, A. et al. Recurrent Glioblastoma Treated with Recombinant Poliovirus. $N$ Engl J Med 379, 150-161, doi:10.1056/NEJMoa1716435 (2018).

41 Markert, J. M. et al. A phase 1 trial of oncolytic HSV-1, G207, given in combination with radiation for recurrent GBM demonstrates safety and radiographic responses. Mol Ther 22, 1048-1055, doi:10.1038/mt.2014.22 (2014).

42 Brown, C. E. et al. Bioactivity and Safety of IL13Ralpha2-Redirected Chimeric Antigen Receptor CD8+ T Cells in Patients with Recurrent Glioblastoma. Clin Cancer Res 21, 4062-4072, doi:10.1158/1078-0432.CCR-15-0428 (2015).

43 Ahmed, N. et al. HER2-Specific Chimeric Antigen Receptor-Modified Virus-Specific T Cells for Progressive Glioblastoma: A Phase 1 Dose-Escalation Trial. JAMA Oncol 3, 1094-1101, doi:10.1001/jamaoncol.2017.0184 (2017).

44 O'Rourke, D. M. et al. A single dose of peripherally infused EGFRvIII-directed CAR T cells mediates antigen loss and induces adaptive resistance in patients with recurrent glioblastoma. Sci Transl Med 9, doi:10.1126/scitranslmed.aaa0984 (2017).

45 Ott, P. A. et al. T-Cell-Inflamed Gene-Expression Profile, Programmed Death Ligand 1 Expression, and Tumor Mutational Burden Predict Efficacy in Patients Treated With Pembrolizumab Across 20 Cancers: KEYNOTE-028. J Clin Oncol 37, 318-327, doi:10.1200/JCO.2018.78.2276 (2019). 
61447 Holmgaard, R. B. et al. Tumor-Expressed IDO Recruits and Activates MDSCs in a Treg-

615 Dependent Manner. Cell Rep 13, 412-424, doi:10.1016/j.celrep.2015.08.077 (2015).

61648 Croft, M., So, T., Duan, W. \& Soroosh, P. The significance of OX40 and OX40L to Tcell biology and immune disease. Immunol Rev 229, 173-191, doi:10.1111/j.1600065X.2009.00766.x (2009).

Interfering with Interleukin-2 Signaling Early during HIV-1 Infection. J Virol 90, 79677979, doi:10.1128/JVI.00994-16 (2016).

50 Sharma, P. \& Allison, J. P. The future of immune checkpoint therapy. Science 348, 5661, doi:10.1126/science.aaa8172 (2015).

51 Cao, S. et al. Dynamic host immune response in virus-associated cancers. Commun Biol 2, 109, doi:10.1038/s42003-019-0352-3 (2019).

52 Vigano, S., Perreau, M., Pantaleo, G. \& Harari, A. Positive and negative regulation of cellular immune responses in physiologic conditions and diseases. Clin Dev Immunol 2012, 485781, doi:10.1155/2012/485781 (2012).

53 Goldszmid, R. S., Dzutsev, A. \& Trinchieri, G. Host immune response to infection and cancer: unexpected commonalities. Cell Host Microbe 15, 295-305, doi:10.1016/j.chom.2014.02.003 (2014).

54 Wykes, M. N. \& Lewin, S. R. Immune checkpoint blockade in infectious diseases. Nature reviews. Immunology 18, 91-104, doi:10.1038/nri.2017.112 (2018).

55 Yoshida, R., Urade, Y., Tokuda, M. \& Hayaishi, O. Induction of indoleamine 2,3dioxygenase in mouse lung during virus infection. Proceedings of the National Academy of Sciences of the United States of America 76, 4084-4086 (1979).

56 Giovannoni, F., Li, Z., Garcia, C. C. \& Quintana, F. J. A potential role for AHR in SARS-CoV-2 pathology. Res $S q$, rs.3.rs-25639, doi:10.21203/rs.3.rs-25639/v1 (2020). Terness, P. et al. Inhibition of allogeneic $\mathrm{T}$ cell proliferation by indoleamine 2,3dioxygenase-expressing dendritic cells: mediation of suppression by tryptophan metabolites. J Exp Med 196, 447-457 (2002).

58 Fallarino, F. et al. T cell apoptosis by tryptophan catabolism. Cell death and differentiation 9, 1069-1077, doi:10.1038/sj.cdd.4401073 (2002).

59 Frumento, G. et al. Tryptophan-derived catabolites are responsible for inhibition of T and natural killer cell proliferation induced by indoleamine 2,3-dioxygenase. J Exp Med 196, 459-468 (2002).

60 Takenaka, M. C. et al. Control of tumor-associated macrophages and T cells in glioblastoma via AHR and CD39. Nat Neurosci 22, 729-740, doi:10.1038/s41593-0190370-y (2019).

61 Perepechaeva, M. L. \& Grishanova, A. Y. The Role of Aryl Hydrocarbon Receptor (AhR) in Brain Tumors. Int J Mol Sci 21, doi:10.3390/ijms21082863 (2020).

62 Zhai, L. et al. The role of IDO in brain tumor immunotherapy. J Neurooncol 123, 395403, doi:10.1007/s11060-014-1687-8 (2015).

63 Berrong, Z. et al. Antigen-Specific Antitumor Responses Induced by OX40 Agonist Are Enhanced by the IDO Inhibitor Indoximod. Cancer Immunol Res 6, 201-208, doi:10.1158/2326-6066.CIR-17-0223 (2018). Transl Med 10, doi:10.1126/scitranslmed.aan4488 (2018). 
65 Jin, G. B., Moore, A. J., Head, J. L., Neumiller, J. J. \& Lawrence, B. P. Aryl hydrocarbon 660 receptor activation reduces dendritic cell function during influenza virus infection. Toxicol Sci 116, 514-522, doi:10.1093/toxsci/kfq153 (2010).

66 Jin, G. B., Winans, B., Martin, K. C. \& Paige Lawrence, B. New insights into the role of the aryl hydrocarbon receptor in the function of $\mathrm{CD} 11 \mathrm{c}(+)$ cells during respiratory viral

67 Lawrence, B. P., Roberts, A. D., Neumiller, J. J., Cundiff, J. A. \& Woodland, D. L. Aryl hydrocarbon receptor activation impairs the priming but not the recall of influenza virusspecific CD8+ T cells in the lung. J Immunol 177, 5819-5828, doi:10.4049/jimmunol.177.9.5819 (2006).

68 Warren, T. K., Mitchell, K. A. \& Lawrence, B. P. Exposure to 2,3,7,8tetrachlorodibenzo-p-dioxin (TCDD) suppresses the humoral and cell-mediated immune responses to influenza A virus without affecting cytolytic activity in the lung. Toxicol Sci 56, 114-123, doi:10.1093/toxsci/56.1.114 (2000).

69 Croft, M. Control of immunity by the TNFR-related molecule OX40 (CD134). Annu Rev Immunol 28, 57-78, doi:10.1146/annurev-immunol-030409-101243 (2010).

70 Grohmann, U., Fallarino, F. \& Puccetti, P. Tolerance, DCs and tryptophan: much ado about IDO. Trends Immunol 24, 242-248, doi:10.1016/s1471-4906(03)00072-3 (2003).

71 Mellor, A. L., Keskin, D. B., Johnson, T., Chandler, P. \& Munn, D. H. Cells expressing indoleamine 2,3-dioxygenase inhibit T cell responses. J Immunol 168, 3771-3776, doi:10.4049/jimmunol.168.8.3771 (2002).

72 Sznurkowski, J. J., Zawrocki, A., Emerich, J., Sznurkowska, K. \& Biernat, W. Expression of indoleamine 2,3-dioxygenase predicts shorter survival in patients with vulvar squamous cell carcinoma (vSCC) not influencing on the recruitment of FOXP3expressing regulatory T cells in cancer nests. Gynecol Oncol 122, 307-312, doi:10.1016/j.ygyno.2011.04.050 (2011).

73 Munn, D. H. et al. Inhibition of T cell proliferation by macrophage tryptophan catabolism. J Exp Med 189, 1363-1372 (1999).

74 Curti, A., Trabanelli, S., Salvestrini, V., Baccarani, M. \& Lemoli, R. M. The role of indoleamine 2,3-dioxygenase in the induction of immune tolerance: focus on hematology. Blood 113, 2394-2401, doi:10.1182/blood-2008-07-144485 (2009).

75 Curti, A. et al. Indoleamine 2,3-dioxygenase-expressing leukemic dendritic cells impair a leukemia-specific immune response by inducing potent $\mathrm{T}$ regulatory cells. Haematologica 95, 2022-2030, doi:10.3324/haematol.2010.025924 (2010).

76 Levina, V., Su, Y. \& Gorelik, E. Immunological and nonimmunological effects of indoleamine 2,3-dioxygenase on breast tumor growth and spontaneous metastasis formation. Clin Dev Immunol 2012, 173029, doi:10.1155/2012/173029 (2012).

77 Platten, M. et al. Treatment of autoimmune neuroinflammation with a synthetic tryptophan metabolite. Science 310, 850-855, doi:10.1126/science.1117634 (2005). 


\section{Acknowledgments}

The authors thank Dr. Inder M. Verma (The Salk Institute for Biological Studies, La Jolla, CA, USA) for generously providing the GSC-005 glioma culture; Verlene Henry for providing technical assistance with animal experiments; Drs. William K. Russel and Rahul Deshpande (Mass Spectrometry Facility, University of Texas Medical Branch at Galveston, TX, USA) for performing the liquid chromatography-mass spectrometry analysis; and Joseph Munch, Mark Picus, and Stephanie Deming (Editing Services in MD Anderson Cancer Center Research Medical Library) for their assistance in language editing of this manuscript.

This work was supported by the National Institutes of Health/National Cancer Institute (NIH/NCI) F31CA228207 (T.T.N.), R01CA256006 (J.F., C.G.-M.), P50CA127001 (J.F., F.F.L.), and U54CA096297 (C.G.-M., F.G.-V.); James P. Harris Brain Tumor Research Fund; Bradley Zankel Foundation (J.F.); and John and Rebekah Harper Fellowship (D.H.S.). This study also used MD Anderson's Research Animal Support Facility and Advanced Technology Genomics Core, which are supported in part by the NIH/NCI through MD Anderson's Cancer Center Support Grant P30CA01667, and the Cancer Prevention and Research Institute of Texas (CPRIT) RP190682 supporting the Mass Spectrometry Facility at the University of Texas Medical Branch, Galveston, TX. The funding bodies were not involved in the study design, the data collection and analysis, the decision to publish, or the preparation of the manuscript.

\section{Author contributions}

Conceptualization and design: T.T.N., H.J., F.F.L., M.M.A., D.A.W., C.G.-M., J.F. Development of methodology and acquisition of data: T.T.N., D.H.S., S.S., Y.R.-M., X.F., J.G., L.Z., E.L., K.L.L. Data analysis: T.T.N., S.K.S., H.J., S.S., C.A.-B., F.G.V. Supervision: C.G.-M, J.F. Writing, review, and/or revision of the manuscript: T.T.N., C.G.-M., J.F.

\section{Competing interests}

M.M.A., H.J., F.F.L., C.G.-M., and J.F. report license agreements with DNAtrix, Inc. C.G.-M. and J.F. are founders of DNAtrix, Inc. MMA reports DNAtrix-sponsored research not related to this work.

\section{Additional information}

Supplementary information is submitted with this manuscript. 
Figure 1: Delta-24-RGDOX treatment remodels the tumor microenvironment.

A

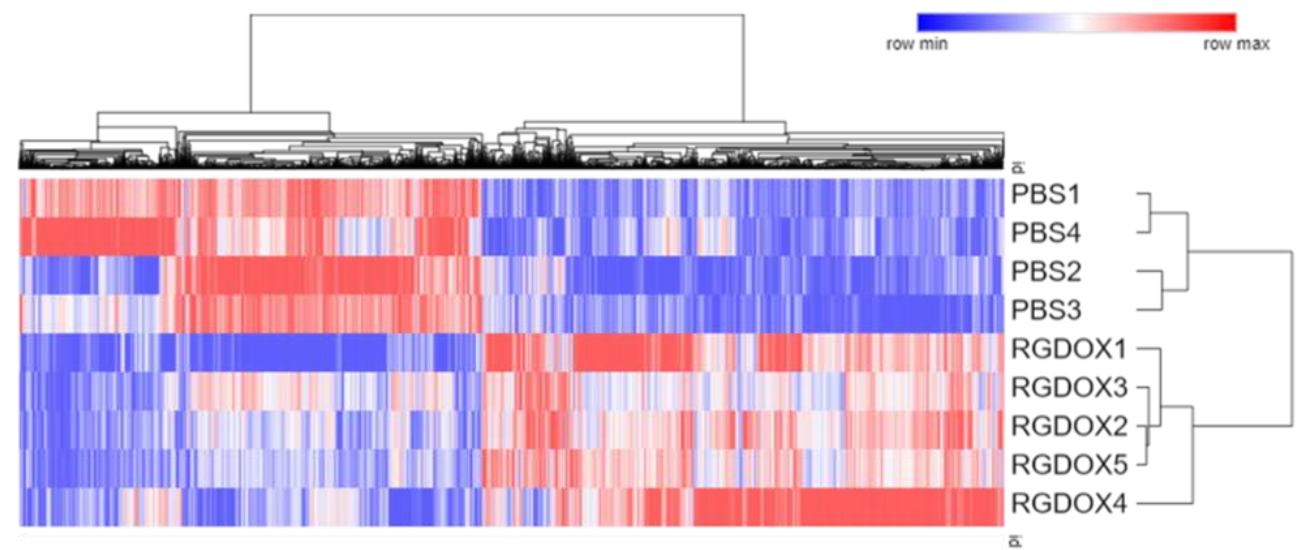

B

GO: Enrichment (PBS vs. RGDOX)

\begin{tabular}{|c|c|c|c|c|}
\hline GO biological process complete & Number & overUnder $^{*}$ & pvalue & FDR \\
\hline positive regulation of cell adhesion (GO:0045785) & 207 & - & $0.00 \mathrm{E}+00$ & $0.00 \mathrm{E}+00$ \\
\hline humoral immune response (GO:0006959) & 64 & - & $0.00 \mathrm{E}+00$ & $0.00 \mathrm{E}+00$ \\
\hline immune response (GO:0006955) & 505 & - & $0.00 \mathrm{E}+00$ & $0.00 \mathrm{E}+00$ \\
\hline inflammatory response (GO:0006954) & 219 & - & $0.00 \mathrm{E}+00$ & $0.00 \mathrm{E}+00$ \\
\hline defense response (GO:0006952) & 528 & - & $0.00 \mathrm{E}+00$ & $0.00 \mathrm{E}+00$ \\
\hline nervous system development (GO:0007399) & 785 & + & $0.00 \mathrm{E}+00$ & $0.00 \mathrm{E}+00$ \\
\hline mitotic cell cycle process (GO:1903047) & 208 & + & $0.00 \mathrm{E}+00$ & $0.00 \mathrm{E}+00$ \\
\hline cell cycle (GO:0007049) & 467 & + & $0.00 \mathrm{E}+00$ & $0.00 \mathrm{E}+00$ \\
\hline microtubule-based process (GO:0007017) & 269 & + & $0.00 \mathrm{E}+00$ & $0.00 \mathrm{E}+00$ \\
\hline cell division (GO:0051301) & 211 & + & $0.00 \mathrm{E}+00$ & $0.00 \mathrm{E}+00$ \\
\hline & & & \\
\hline
\end{tabular}

C

Canonical Pathways (PBS vs. RGDOX)

T Cell Receptor Signaling ${ }^{\dagger}$ Hypercytokinemia/hyperc.. TREM1 Signaling IL-17 Signaling ${ }^{\dagger \dagger}$ Neuroinflammation...

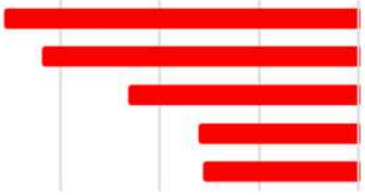

$$
\begin{array}{llll}
-5.8 & -5.6 & -5.4 & -5.2
\end{array}
$$

Activation z-Score

\section{$\mathbf{E}$}

IDO Network: PBS vs. RGDOX

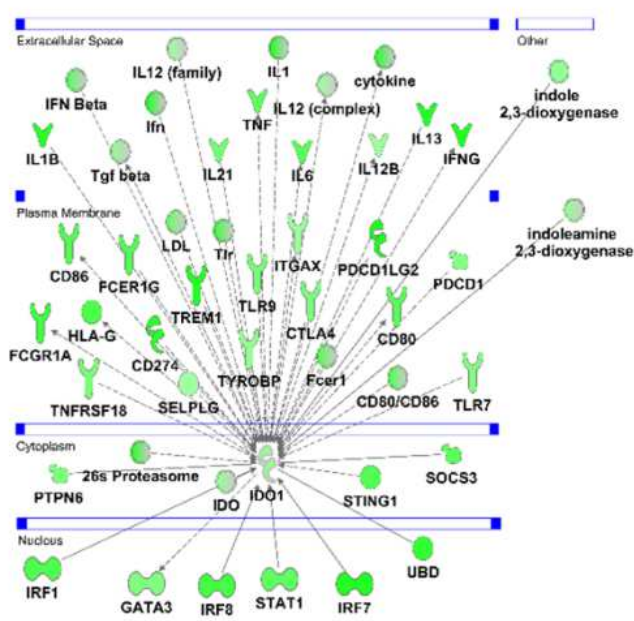

Upstream Regulator (PBS vs. RGDOX)

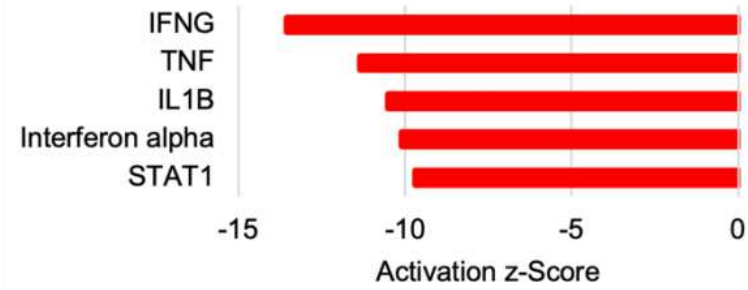

$\mathbf{F}$

Prediction of immune Cell Composition

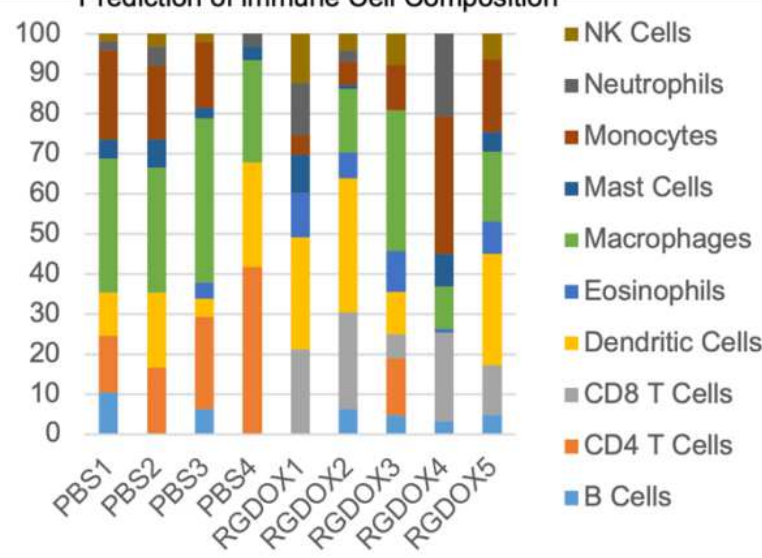


Fig. 1. Delta-24-RGDOX treatment remodels the tumor microenvironment. Differentially expressed genes in tumors treated with PBS versus tumors treated with Delta-24-RGDOX were utilized for clustering, gene ontology (GO) biological process enrichment analysis, and pathway and network analyses. (A) Heatmap comparing the transcriptional signatures of intracranial GL261-5-derived tumors treated with PBS or Delta-24-RGDOX. The log2-normalized expression levels of genes with significant adjusted $P$-values $(<0.05)$ across samples are shown. The color scale is shown above the heatmap. (B) GO biological process enrichment results for tumors treated with PBS or Delta-24-RGDOX. The five most significant GO biological processes are shown. GO biological processes significantly associated with PBS-treated tumors are marked as '+', whereas those significantly associated with Delta-24-RGDOX-treated tumors are marked as ' - '. (C) The five most significantly altered canonical pathways in tumors treated with PBS versus Delta-24-RGDOX. Activation z-scores are plotted in the graph. ${ }^{\dagger}$ Hypercytokinemia/hyperchemokinemia in the pathogenesis of influenza; †ंneuroinflammation signaling pathway. (D) The five most significantly altered upstream regulators in tumors treated with PBS versus Delta-24-RGDOX. Activation z-scores are plotted in the graph. The negative zscores represent activation in Delta-24-RGDOX. (E) IDO1 network genes with significantly altered expression levels in tumors treated with PBS or Delta-24-RGDOX. The color intensity indicates the $\log 2$ fold-change (green $=$ activation) levels for each gene in PBS-treated tumors versus Delta-24-RGDOX-treated tumors. (F) The prediction of the immune cell composition in GL261-5-derived brain tumors treated with PBS or Delta-24-RGDOX. The percentages of various immune cell populations in each sample are presented in the graph. The color code for 
Figure 2: Oncolytic adenoviruses induce the expression and activation of the IDO-Kyn-AhR cascade in vivo and in vitro.
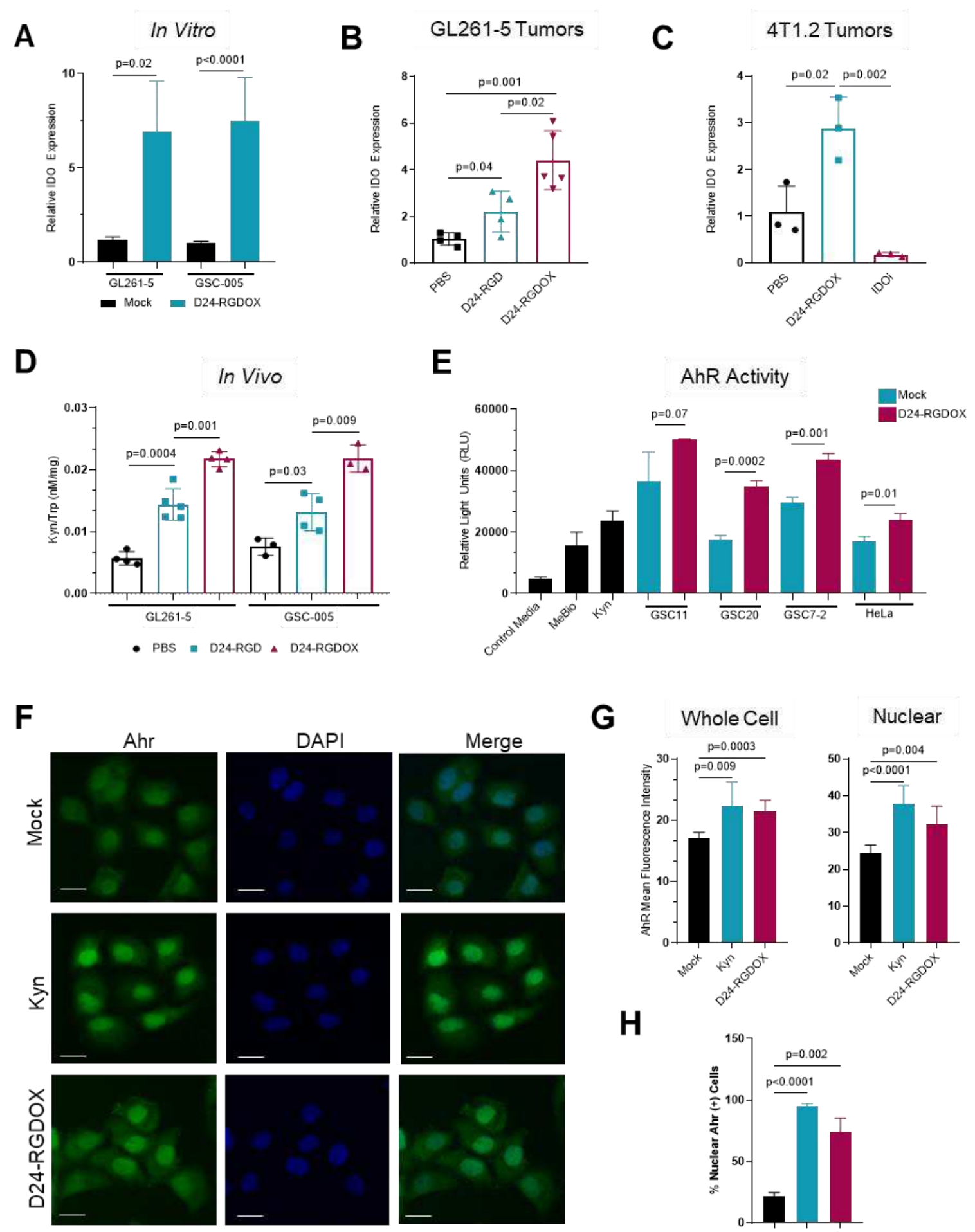

H

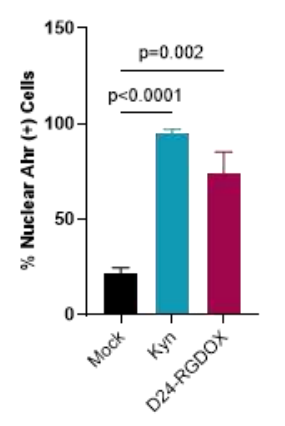


Fig. 2. Oncolytic adenoviruses induce the expression and activation of the IDO-Kyn-AhR cascade in vivo and in vitro. (A) IDO expression in GL261-5 and GSC-005 murine glioma cells in response to Delta-24-RGDOX infection. Cells were infected with Delta-24-RGDOX (100-150 multiplicities of infection [MOIs]) over $48 \mathrm{~h}$. RNA was extracted, and the relative levels of IDO were measured using qRT-PCR; GAPDH or $\beta$-Actin was used as a housekeeping gene control. The column graph shows $2^{\wedge}\left(\mathrm{Ct}^{\mathrm{GAPDH}}\right.$ or $\beta$-Actin-Ct $\left.{ }^{\mathrm{IDO}}\right)$ results normalized to the mock-infected control. (B and C) Relative IDO expression in GL261-5 or 4T1.2 tumors in response to oncolytic adenovirus treatment in vivo. In (B and/or D), the mice were implanted intracranially with GL261-5 or GSC-005 cells and intratumorally treated with PBS, Delta-24-RGD, or Delta-24RGDOX; brain tumors were collected and flash-frozen on day 12. In (C), the mice were implanted with 4T1.2 cells in the right mammary fat pad and treated with PBS, D24-RGDOX, or indoximod (IDOi); tumors were collected and flash-frozen on day 36. RNA was extracted and analyzed as described in (A). (D) The Kyn and Trp metabolite levels in murine gliomas following the indicated treatments (described in $[\mathrm{B}]$ ) were quantified using liquid chromatography-mass spectrometry. Data are presented as the ratios \pm SDs of Kyn concentrations to Trp concentrations. (E) AhR activity in human GSC lines and HeLa cells in response to Delta-24-RGDOX infection. Cells were mock-infected or infected with Delta-24RGDOX (50 MOI) over $48 \mathrm{~h}$, and the transcriptional activity of AhR in the cells was quantified by evaluating the supernatants. Controls included medium (negative), medium containing the AhR agonist MeBio (0.32 nM), and medium containing Kyn $(25 \mu \mathrm{M})$. HeLa cells were used as a positive control. (F) AhR expression and nuclear translocation upon Delta-24-RGDOX treatment. HeLa cells were infected with Delta-24-RGDOX (25 MOI) over $48 \mathrm{~h}$ or treated with Kyn (positive control) and then immunostained for AhR detection. Representative images of AhR-FITC staining, DAPI (nuclear) staining, and merged FITC/DAPI staining are shown. Scale bars, $50 \mu \mathrm{m}$. (G) Quantification of the mean fluorescence intensity of AhR in whole cells and nuclear compartments. (H) Frequency of nuclear AhR-positive cells after the indicated treatments, as analyzed using ImageJ software. Data are shown as the means \pm SDs. P-values were generated by using a two-tailed Student's t-test. 
Figure 3: The viral activity of Delta-24-RGDOX is preserved in the context of IDO inhib7în.

A

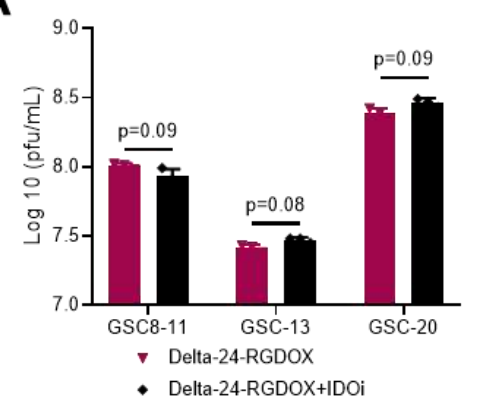

C

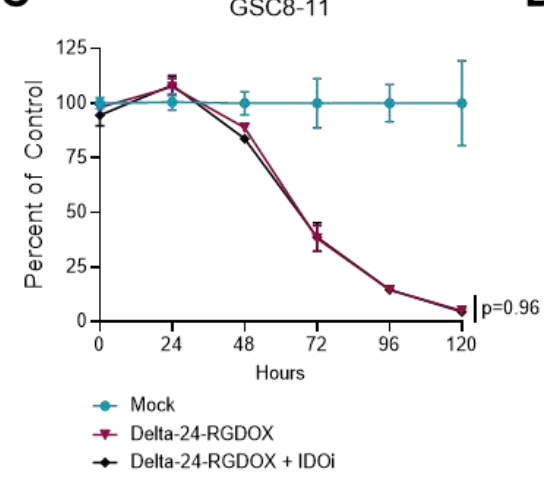

B

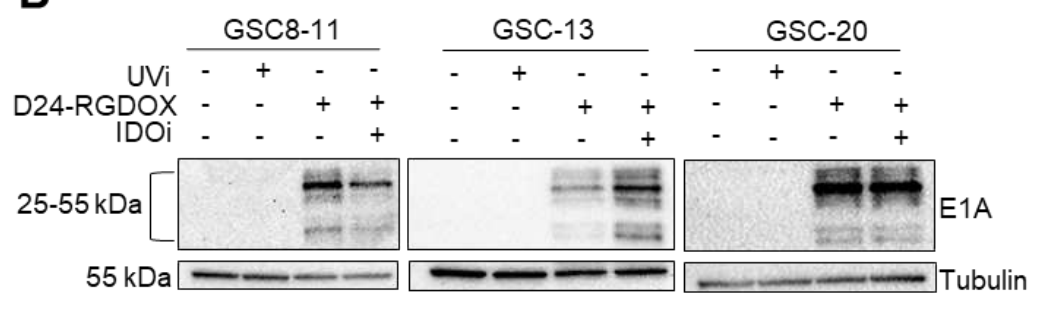

D

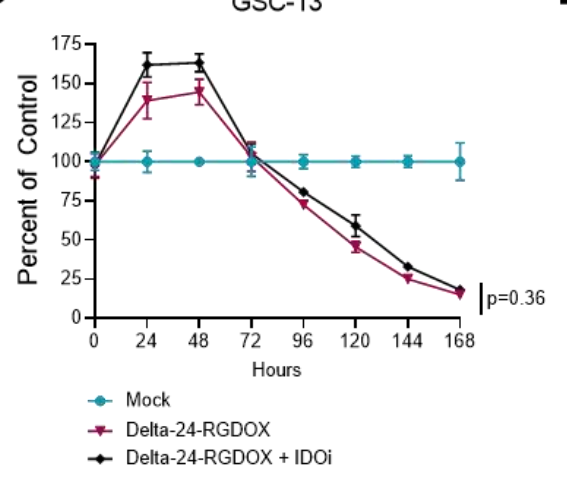

E

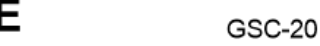

$\mathbf{F}$

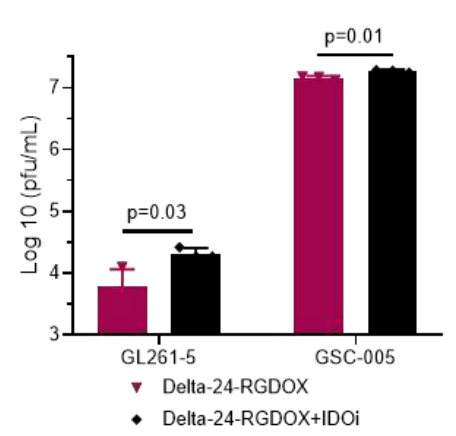

G
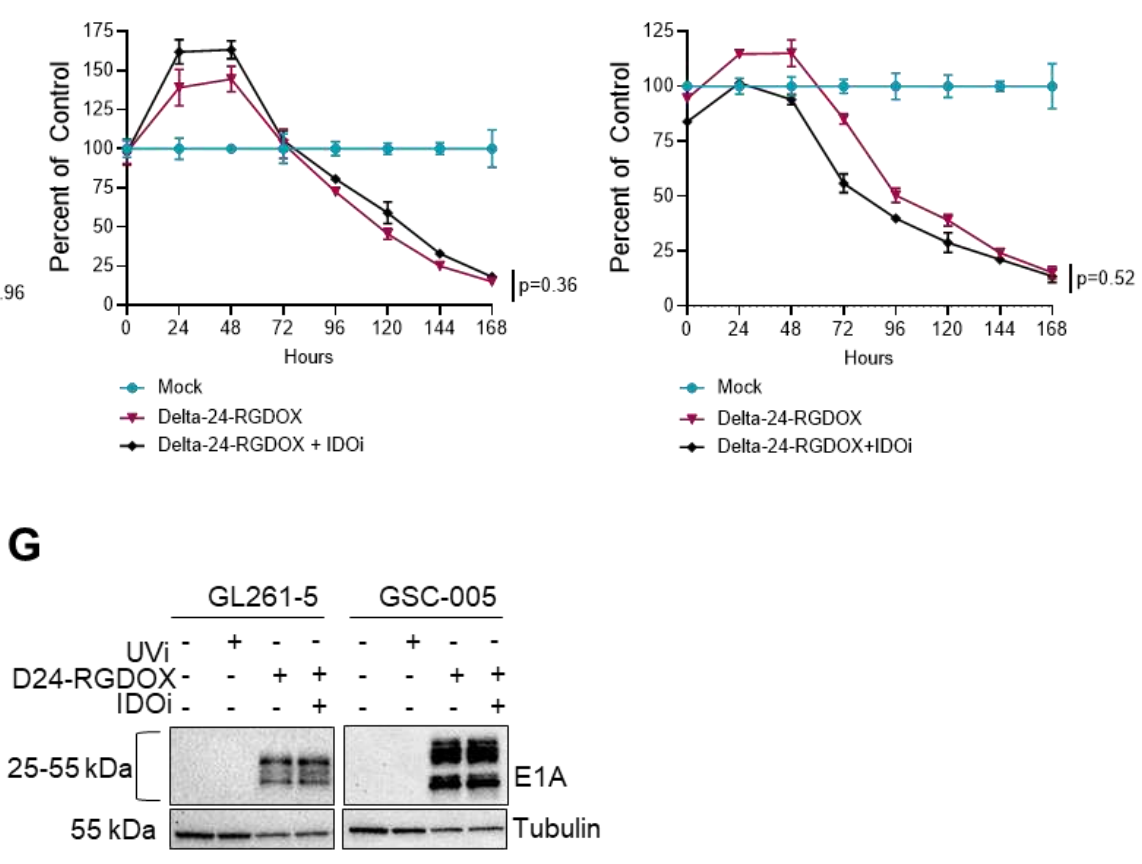

$\rightarrow$ Mock

$\rightarrow$ Delta-24-RGDOX

$\rightarrow$ Delta-24-RGDOX+1DOi
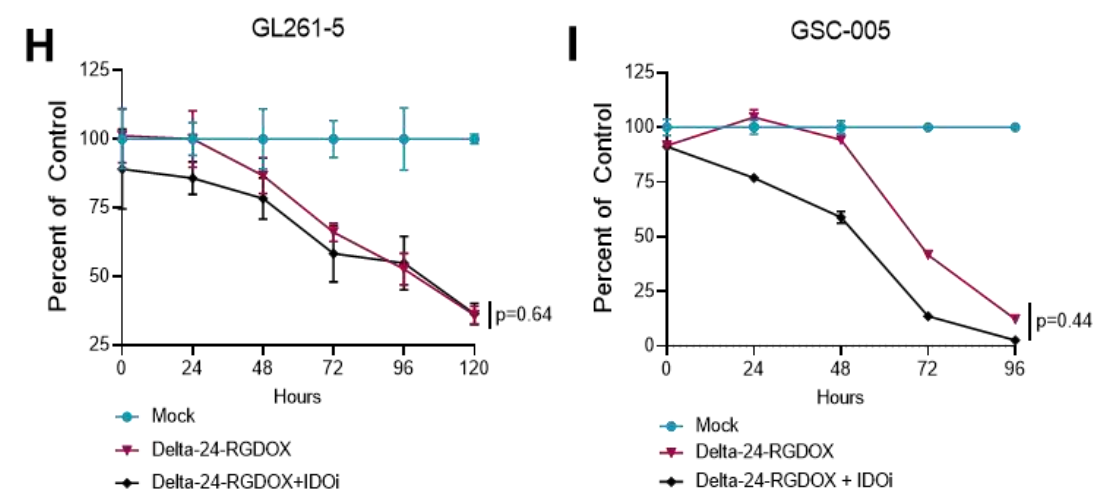

- Mock

* Delta-24-RGDOX

$\rightarrow$ Delta-24-RGDOX + IDOi 
793 Fig. 3. The viral activity of Delta-24-RGDOX is preserved in the context of IDO inhibition.

794 (A) Viral replication of Delta-24-RGDOX in human GSCs with or without an IDO inhibitor

795 (IDOi). The viral concentration (pfu/ml) at $48 \mathrm{~h}$ after cell infection with Delta-24-RGDOX (25

796 MOI) alone or in combination with an IDOi (BGB-7204; $150 \mathrm{nM}$ ) was determined using a hexon

797 titration assay performed with HEK293 cells. Initial viral input: $\log _{10}(6.57) \mathrm{pfu} / \mathrm{ml}$. (B)

798 Expression of the viral protein E1A in whole-cell lysates of human GSCs at $48 \mathrm{~h}$ after treatment

799 with Delta-24-RGDOX (25 MOI) with or without an IDOi (BGB-7204; $150 \mathrm{nM}$ ), as assessed by

800 Western blotting. Tubulin was used as a loading control. UVi, UV-inactivated Delta-24-RGDOX.

801 (C-E) Viability of human GSCs infected with Delta-24-RGDOX (25 MOI) in the presence or

802 absence of an IDOi (BGB-7204; $150 \mathrm{nM}$ ). (F) Viral replication of Delta-24-RGDOX at $48 \mathrm{~h}$

803 after infection of murine glioma cells (100-150 MOIs) with or without treatment with an IDOi

804 (BGB-7204; $50 \mathrm{nM}$ ), as explained in (A). Initial viral input: $\log _{10}(7.18)$ to $\log _{10}(7.35) \mathrm{pfu} / \mathrm{ml}$.

805 (G) Expression of the viral protein E1A in murine glioma cells at $48 \mathrm{~h}$ after treatment with Delta-

806 24-RGDOX (100-150 MOI) alone or in combination with an IDOi (BGB-7204; $50 \mathrm{nM}$ ), as

807 explained in (B). (H-I) Viability of murine glioma cells infected with Delta-24-RGDOX (100-

808150 MOIs) and treated with or without an IDOi (BGB-7204; $50 \mathrm{nM}$ ). Data are shown as the

809 means \pm SDs. P-values were derived by using a two-tailed Student's t-test. 
Figure 4: IDO inhibition modulates the tumor microenvironment of Delta-24-RGDOX treated murine brain tumors.

A
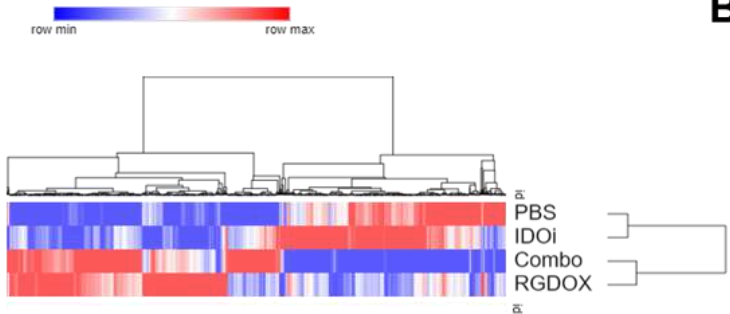

C

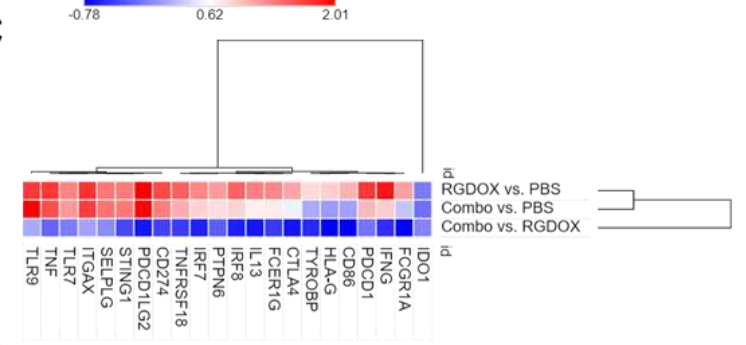

D
B

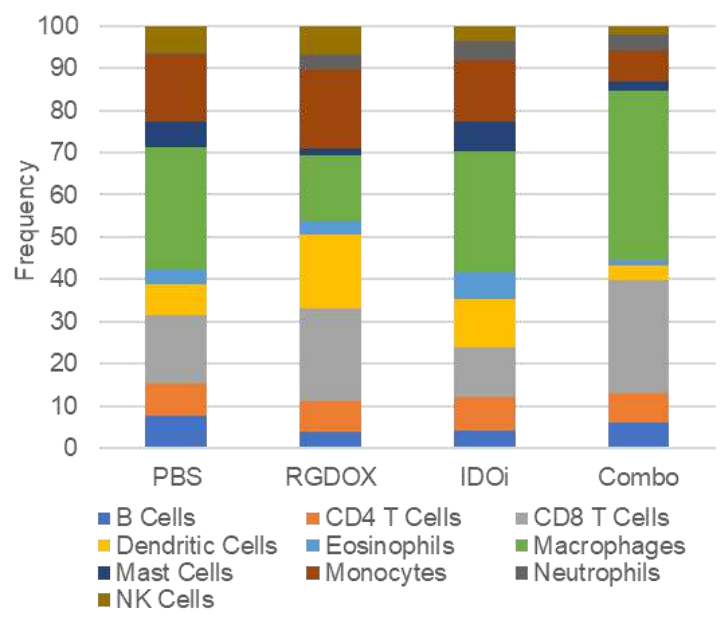

RGDOX vs. PBS

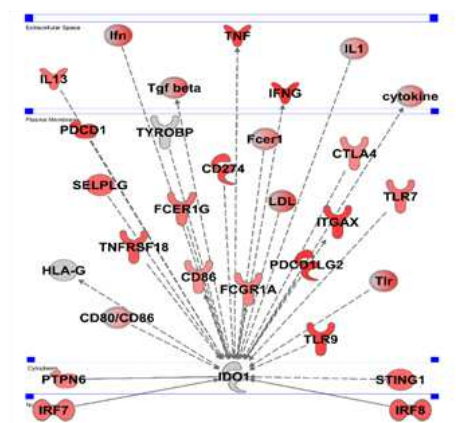

Combovs. PBS

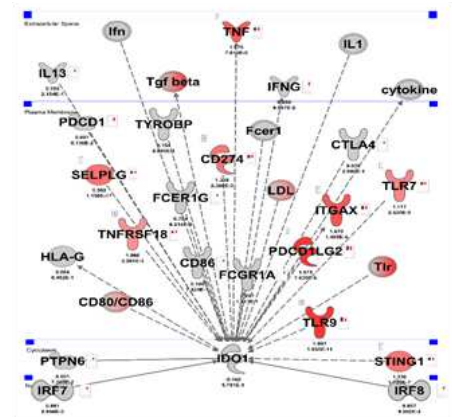

Combovs. RGDOX

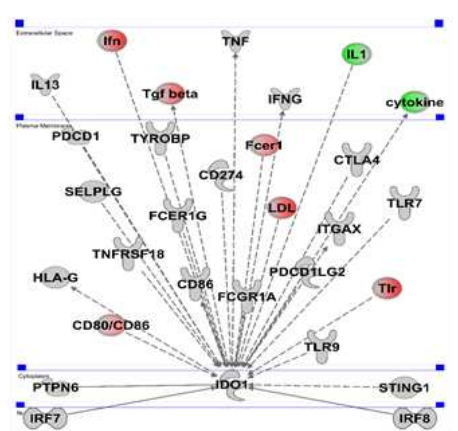


813 Fig. 4. IDO inhibition modulates the tumor microenvironment of Delta-24-RGDOX-treated

814 murine brain tumors. Differentially expressed genes in tumors treated with PBS, the IDO

815 inhibitor (IDOi) indoximod, Delta-24-RGDOX (RGDOX), or the IDOi and Delta-24-RGDOX

816 (combo) utilized for clustering, immune population prediction, and pathway and network

817 analyses. (A) Heatmap comparing the transcriptional signatures of intracranial GL261-5-derived

818 tumors treated with PBS, the IDOi, Delta-24-RGDOX, or the combination therapy. Tumors were

819 established in these mice for 21 days, and the mice underwent treatment for 14 days. The log2-

820 normalized averaged expression levels of genes with significant adjusted $P$-values $(<0.05)$ across

821 sample groups are shown. The color scale is shown above the heatmap. (B) The prediction of the

822 immune cell composition in GL261-5-derived brain tumors in the indicated treatment group.

823 The percentages of various immune cell populations in each sample are presented in the graph.

824 The color code for the various immune cell types is shown at the bottom of the graph. (C)

825 Heatmap or (D) pathway representation of IDO1 network genes with significantly altered

826 expression levels in tumors; treatment group comparisons are indicated. The color scale is shown

827 above the heatmap. The color intensity of the pathway representation graphics indicates the $\log 2$

828 fold-change levels for each gene in the specified treatment group comparison; gray represents

829 unchanged, red represents activation, and green represents inhibition. 
830 Figure 5: Combined treatment with Delta-24-RGDOX and IDO inhibition results in an enhanced therapeutic effect and tumor regression.

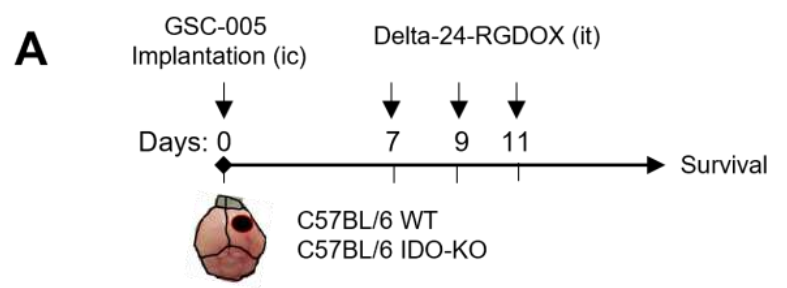

B

C GL261, GSC-005, or B16

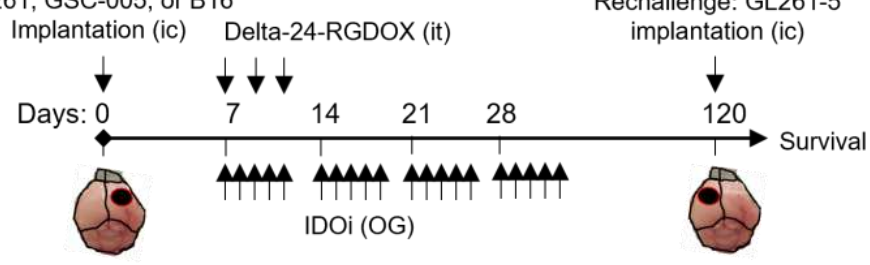

D

GL261-5 Glioma

$E$

Re-challenge
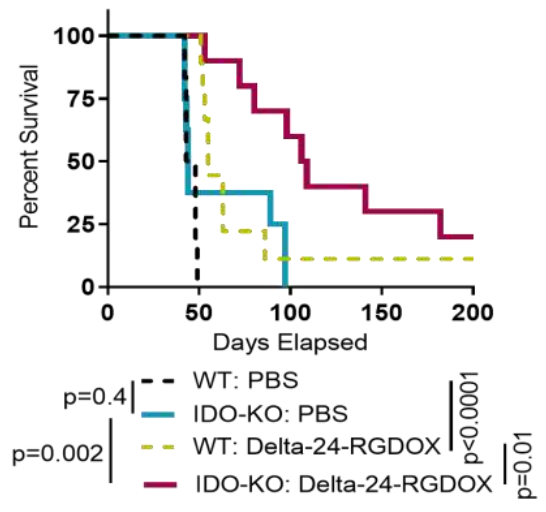

$\mathbf{F}$
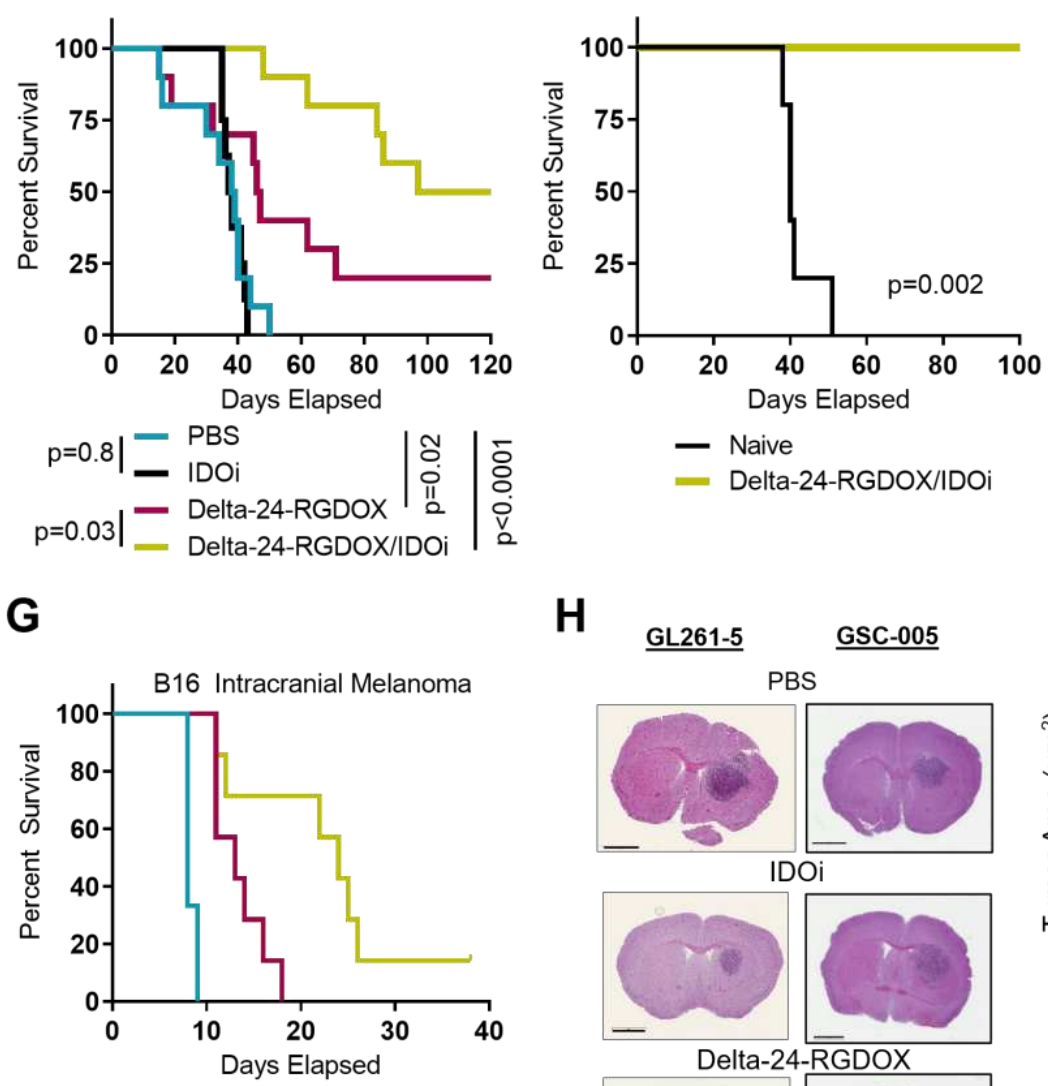

H
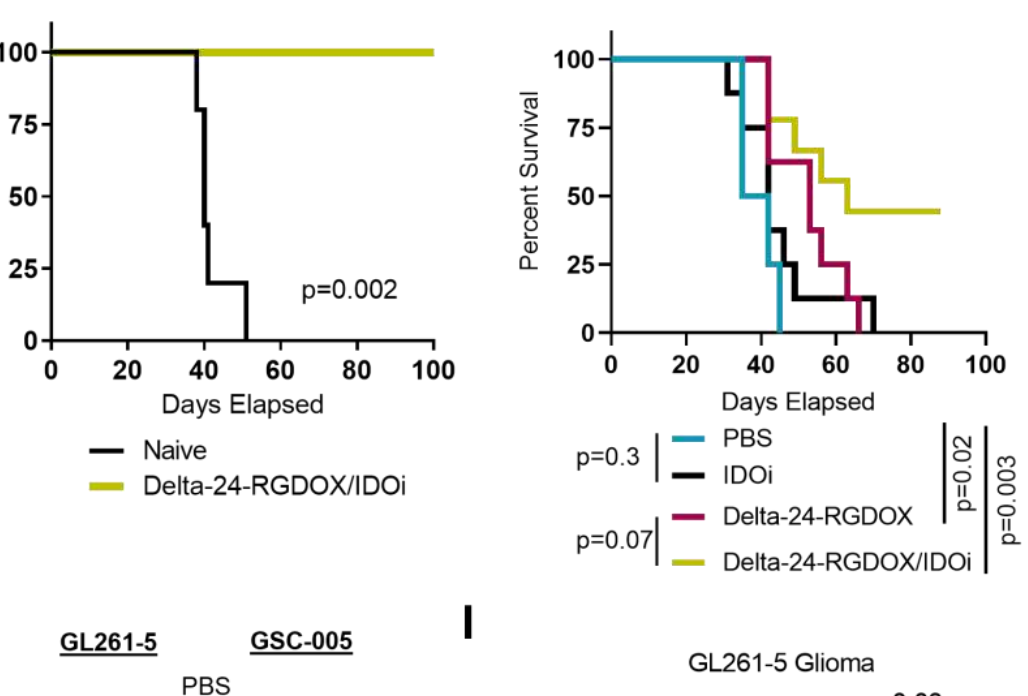

I
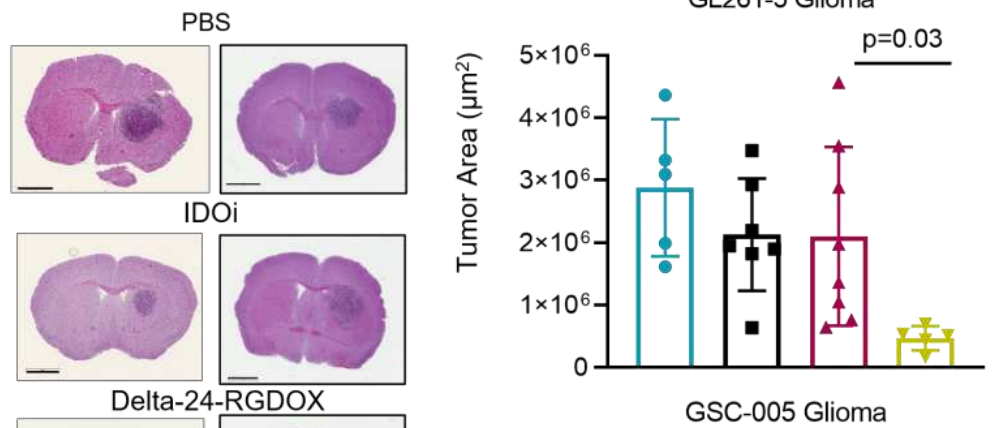

- Control $\quad$ Delta-24-RGDOX $\mid p=0.0003$
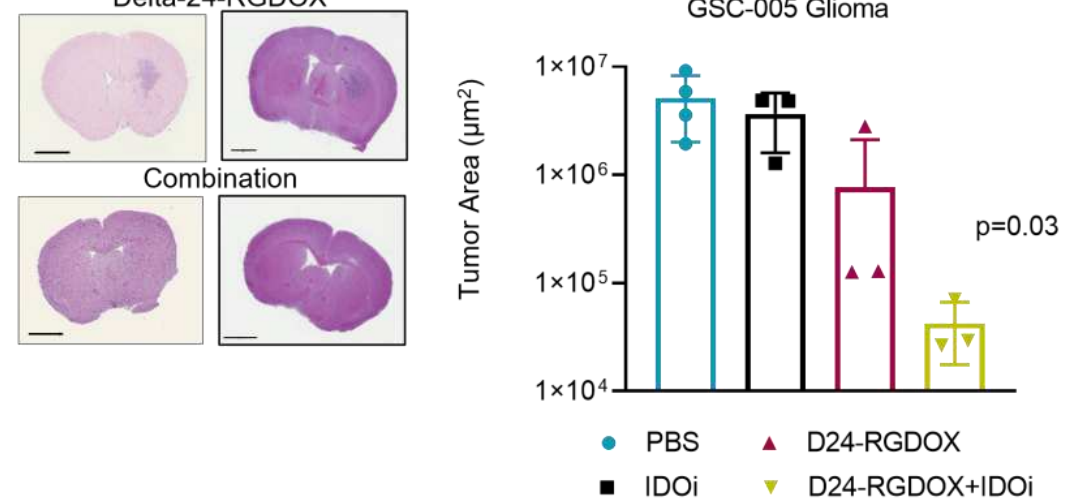
Fig. 5. Combined treatment with Delta-24-RGDOX infection and IDO inhibition results in an enhanced therapeutic effect and tumor regression. (A) Treatment schedule of wild-type (WT) and IDO-KO C57BL/6 mice bearing intracranial (ic) GSC-005 tumors. GSC-005 cells were implanted intracranially (ic) on day 0 , and the mice were randomly assigned to receive intratumoral (it) injection of either Delta-24-RGDOX or vehicle. Survival was monitored for up to 200 days. (B) Kaplan-Meier survival curves of the mice included in the experiment depicted in (A). (C) Treatment schedule of C57BL/6 mice bearing intracranial tumors that were treated with Delta-24-RGDOX alone or in combination with an IDO inhibitor (IDOi). OG, oral gavage. (D) Kaplan-Meier survival curves of intracranial GL261-5 tumor-bearing C57BL/6 mice treated with vehicle or with Delta-24-RGDOX alone or in combination with the IDOi 1-methyl-DLtryptophan (1MT; n=10/group). (E) Long-term survivors previously treated with the combination therapy described in (D) were rechallenged with an intracranial injection of GL216-5 cells into the contralateral hemisphere, and their survival was compared with that of control treatmentnaïve mice (n=5/group). (F) Kaplan-Meier survival curves of intracranial GSC-005 tumorbearing C57BL/6 mice treated with vehicle or with Delta-24-RGDOX alone or in combination with the IDOi BGB-7204 (n=9-10/group). (G) Kaplan-Meier survival curves of intracranial B16 melanoma-bearing C57BL/6 mice treated with PBS or with Delta-24-RGDOX alone or in combination with the IDOi indoximod ( $\mathrm{n}=6$ or 7 per group). ( $\mathbf{H}$ and $\mathbf{I})$ The brains of intracranial GL261-5 or GSC-005 tumor-bearing C57BL/6 mice were subjected to histopathological analyses on day 15 (GL261-5) or 24 (GSC-005) after treatment with PBS, Delta-24-RGDOX, indoximod, or the combination therapy ( $\mathrm{n}=5-8 /$ group). Representative images of H\&E staining $(\mathrm{H})$ and the average tumor surface areas (I) were acquired using Aperio ImageScope software. Scale bar, 2 mm. P-values were derived with the log-rank test (B, D-G), a two-tailed Student's t-test (I), or an ordinary one-way ANOVA (I). 
Figure 6: Combined Delta-24-RGDOX and IDO inhibitor treatment increases intratumoral $T$ cells and decreases immunosuppressive cell populations.

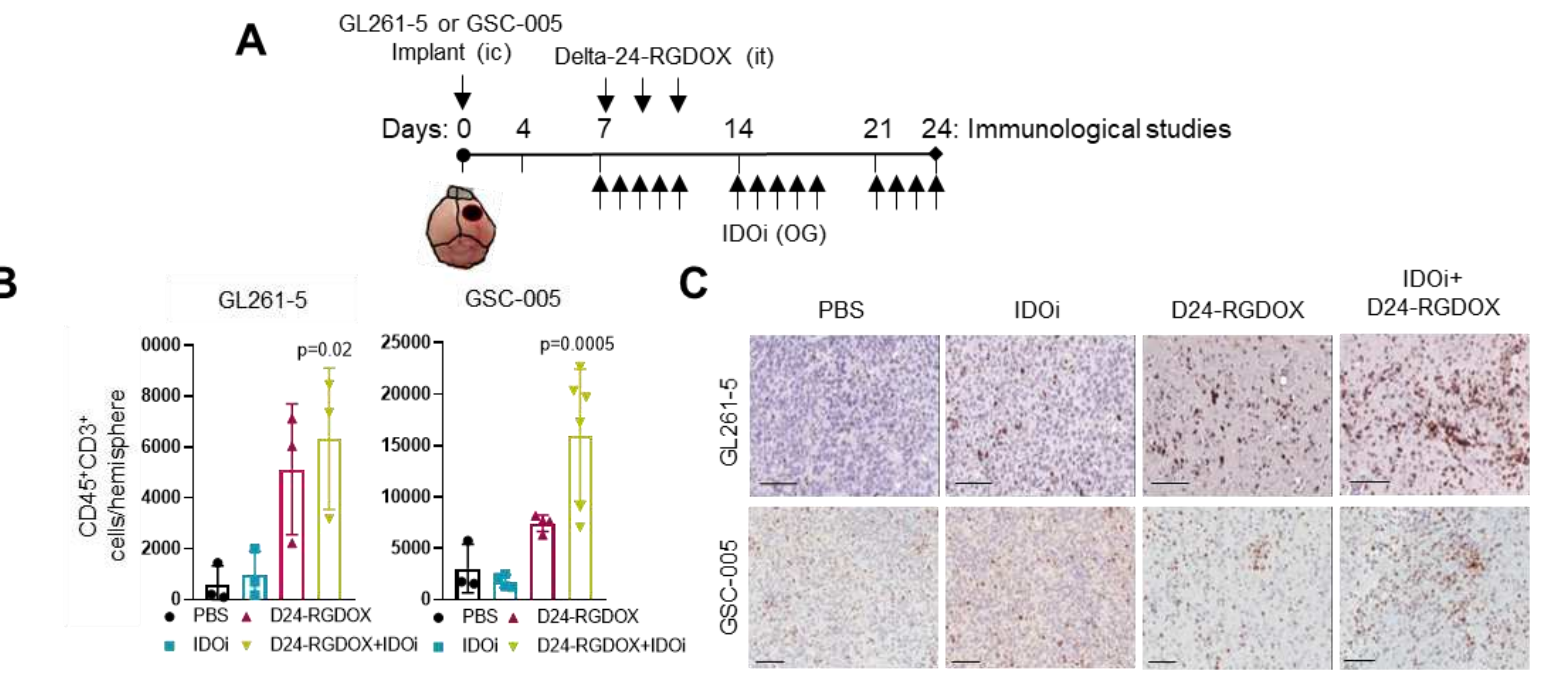

D

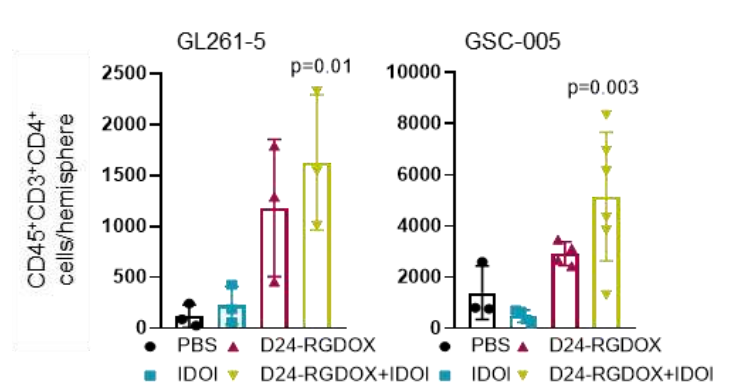

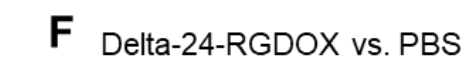

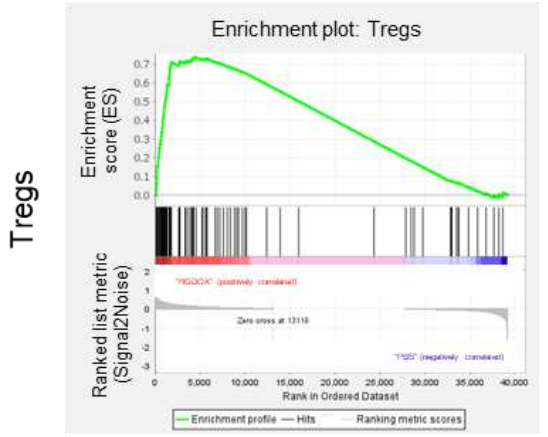

Enrichment plot: MDSCs

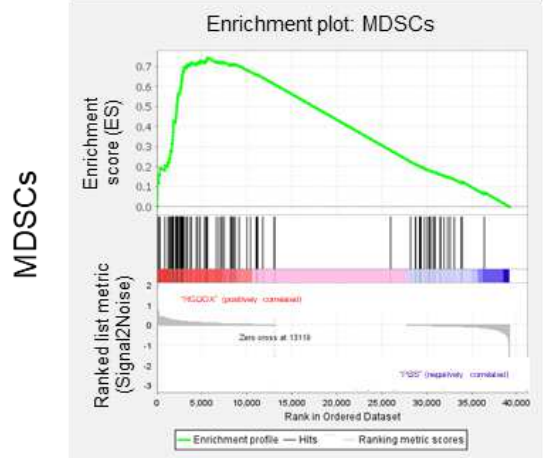

Delta-24-RGDOX vs. Combo
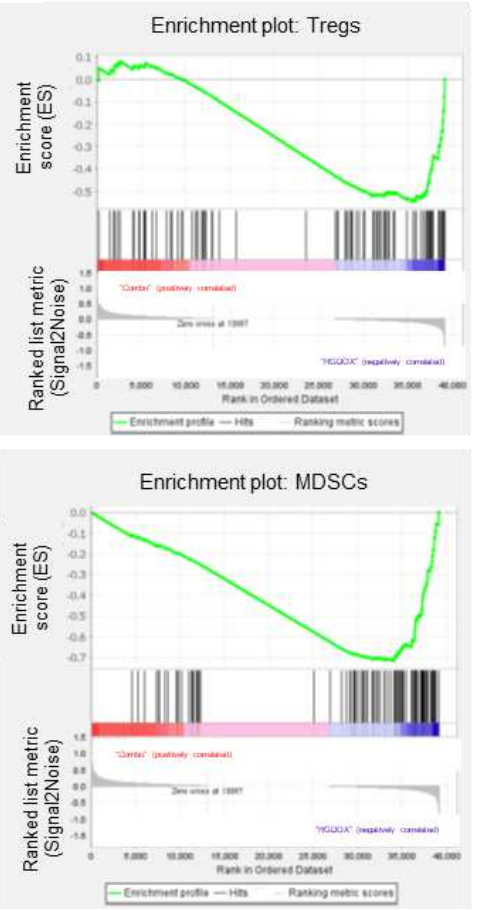

$\mathbf{E}$

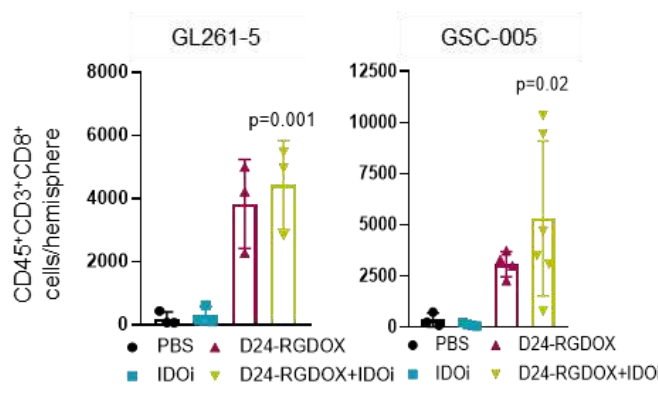

G

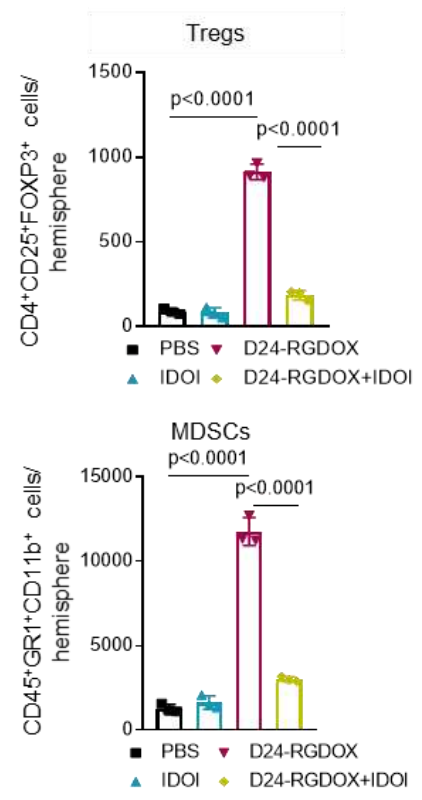


856 Fig. 6. Combined Delta-24-RGDOX and IDO inhibitor treatment increases intratumoral T 857 cells and decreases immunosuppressive cell populations. (A) Treatment timeline for the 858 analysis of immune cell populations. C57BL/6 mice were intracranially (ic) implanted with 859 GL261-5 or GSC-005 cells and randomly assigned to receive PBS (control), an IDO inhibitor 860 (IDOi; GL261-5: indoximod. GSC-005: BGB-7204), Delta-24-RGDOX, or Delta-24-RGDOX 861 plus IDOi. On day 24, brains were collected, stained as indicated, and analyzed using flow 862 cytometry. Parallel experiments were performed for immunohistochemical analyses of the 863 brains. it, intratumorally; OG, oral gavage. (B) Column graphs show the absolute numbers of $864 \mathrm{CD}^{2} 5^{+} \mathrm{CD}^{+}$cells per tumor-containing brain hemisphere in the indicated murine glioma model. 865 (C) Representative CD3 immunohistochemistry images for the indicated treatment groups. 866 Images were acquired using Aperio ImageScope pathology slide viewing software. Scale bar, $867100 \mu \mathrm{m}$. (D and $\mathbf{E}$ ) Column graphs show the absolute numbers of $\mathrm{CD}^{+}(\mathrm{D})$ and $\mathrm{CD} 8^{+}(\mathrm{E}) \mathrm{T}$ 868 cells per hemisphere in the indicated murine glioma models. (F) Enrichment plots for the Treg 869 (top) and MDSC (bottom) gene sets in PBS-treated versus Delta-24-RGDOX-treated or Delta870 24-RGDOX- versus Combo-treated GL261-5 brain tumor RNA. (G) Column graphs show the 871 absolute numbers of $\mathrm{CD} 4^{+} \mathrm{CD} 25^{+} \mathrm{FOXP} 3^{+}$Tregs per hemisphere (top) and $\mathrm{CD} 45^{+} \mathrm{GR} 1{ }^{+} \mathrm{CD} 11 \mathrm{~b}^{+}$ 872 MDSCs per hemisphere (bottom). Data are shown as the means \pm SDs $(n=3)$. P-values were 873 derived with an ordinary one-way ANOVA (B, D-E) or a two-tailed Student's t-test (G). 
Figure 7: Therapeutic efficacy of combined Delta-24-RGDOX infection and IDO inhibition requires $\mathrm{CD4}+\mathrm{T}$-cell activity but not regulatory T-cell activity.

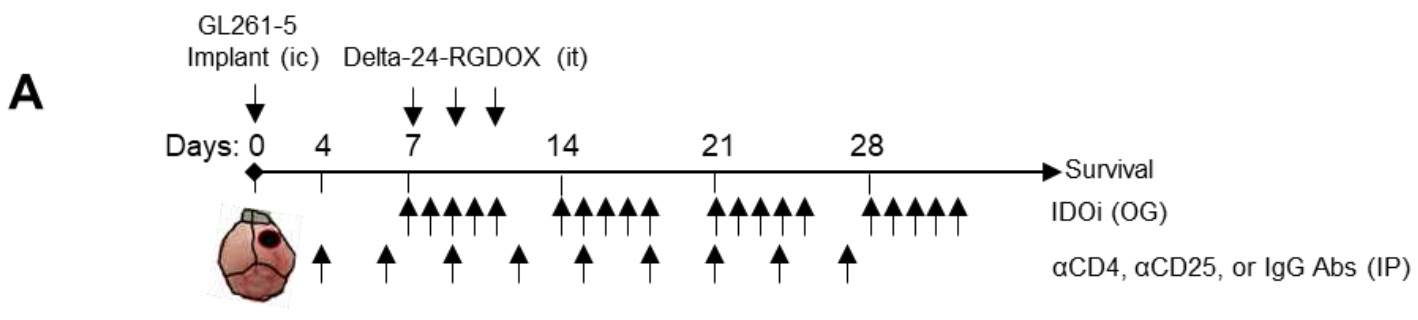

B

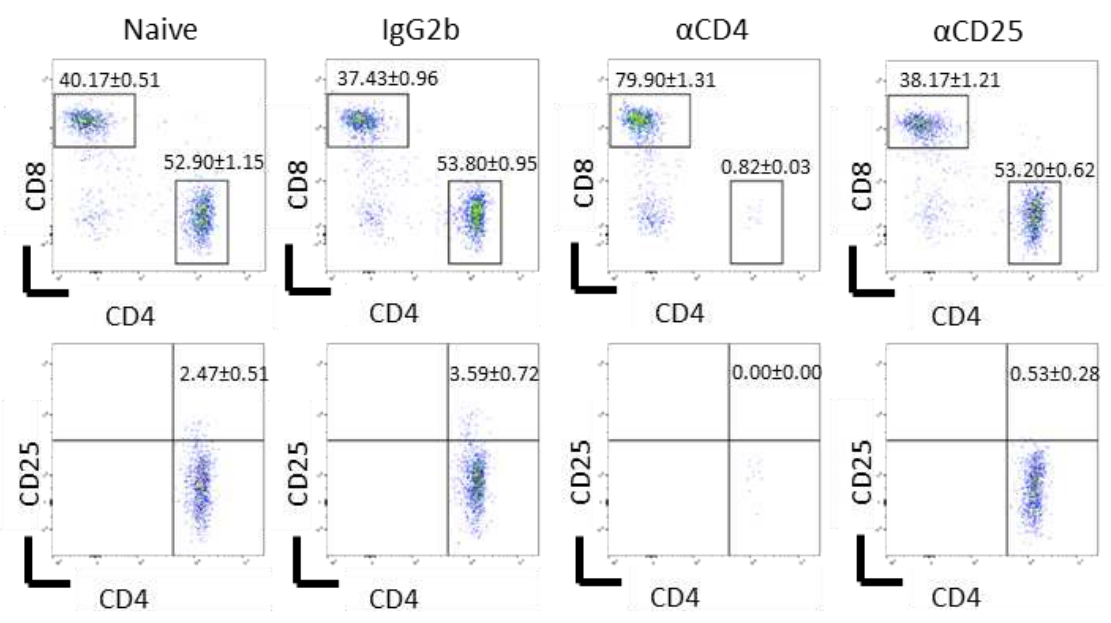

C

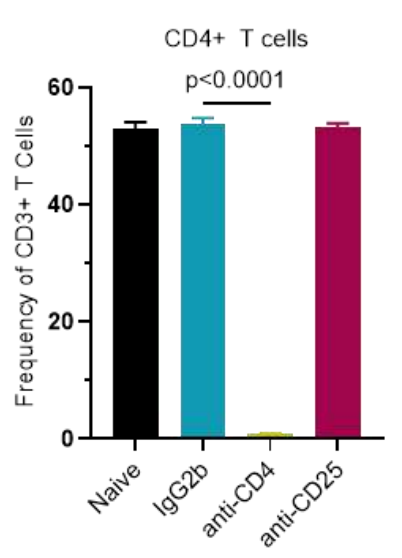

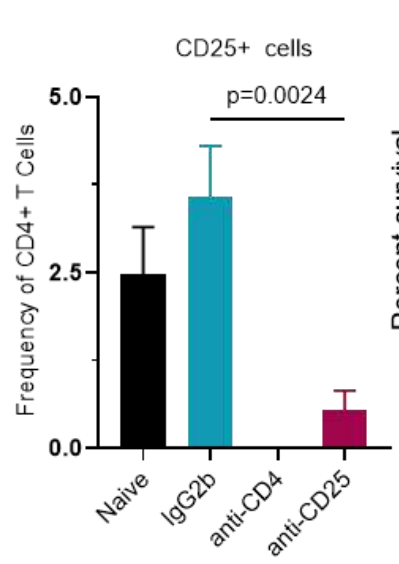

D

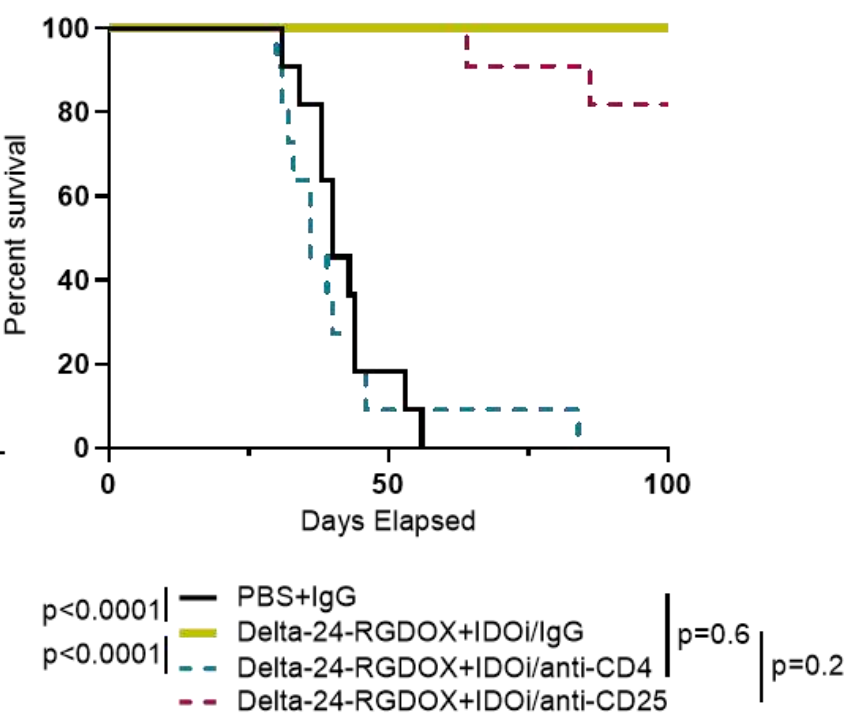


876 Fig. 7. The therapeutic efficacy of combined Delta-24-RGDOX infection and IDO

877 inhibition requires $\mathbf{C D 4}^{+} \mathbf{T}$ cell activity but not Treg activity. (A) Treatment schedule for

878 Delta-24-RGDOX given alone or in combination with an IDO inhibitor (IDOi) in the presence of

879 anti-CD4 or anti-CD25 depleting antibodies. Mice were intracranially (ic) implanted with

880 GL261-5 cells and randomly assigned to receive intratumoral (it) injections of PBS or the

881 combination of Delta-24-RGDOX and the IDOi indoximod ( $\mathrm{n}=10$ or 11 per group, respectively).

882 An IgG control antibody and the anti-CD4 and anti-CD25 depleting antibodies were

883 administered intraperitoneally. OG, oral gavage. (B) Flow cytometry plots showing CD4 ${ }^{+}, \mathrm{CD} 8^{+}$, 884 and $\mathrm{CD} 4{ }^{+} \mathrm{CD} 25^{+} \mathrm{T}$ cell populations in the spleen of treatment-naïve mice and mice treated with

885 IgG2b, anti-CD4, or anti-CD25 at 38 days after tumor implantation. (C) Column graphs show

886 the frequencies of $\mathrm{CD}^{+}$or $\mathrm{CD} 25^{+} \mathrm{T}$ cells in the indicated treatment groups. (D) Kaplan-Meier

887 survival curves of GL261-5 tumor-bearing C57BL/6 mice in the different treatment groups. Data

888 are shown as the means \pm SDs. P-values were derived with a two-tailed Student's t-test (C) or the

889 log-rank test (D).

890 

enhances anti-viral and anti-tumor T-cell activation.
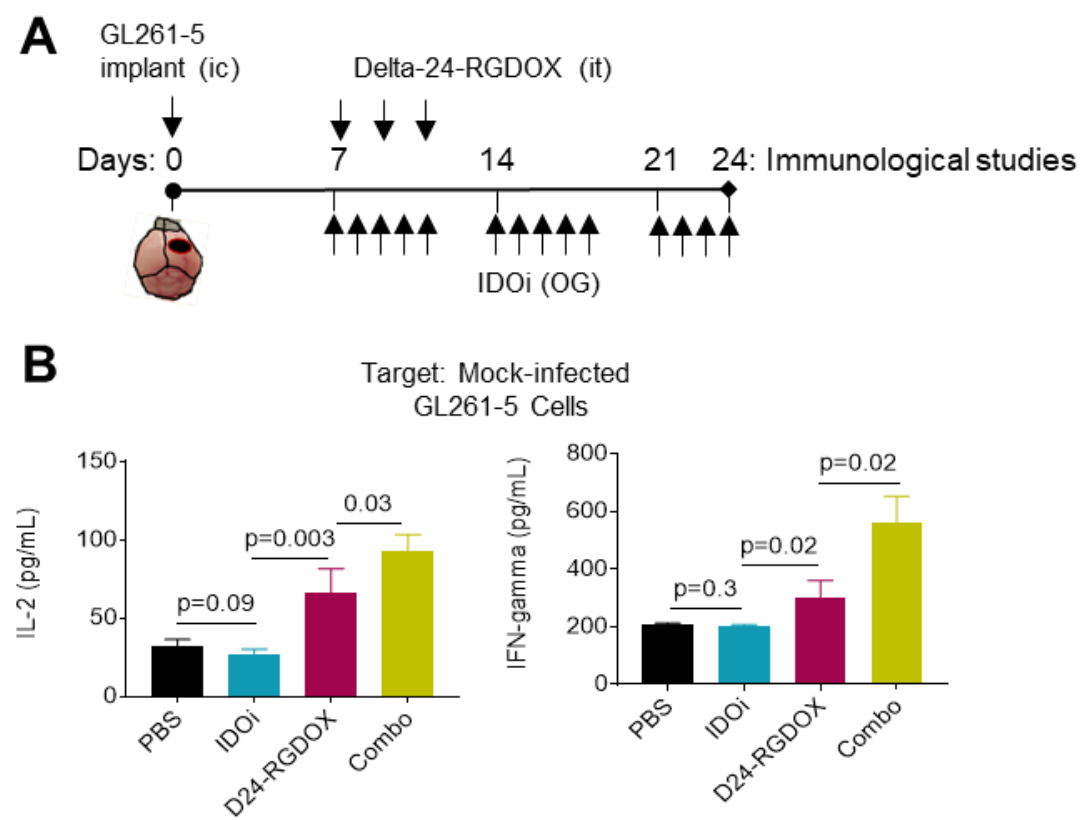

GL261-5 Cells
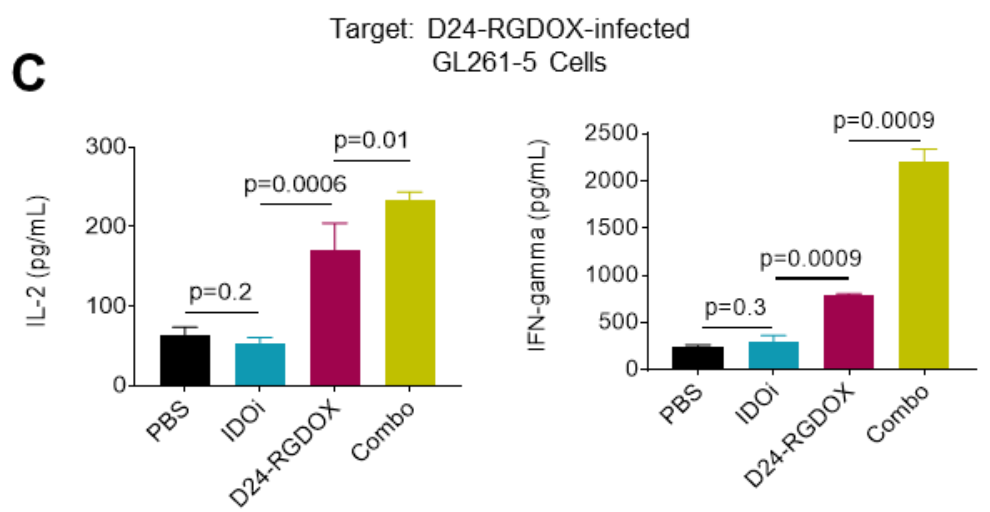

D Target: Mock-infected

Target: D24-RGDOX-infected
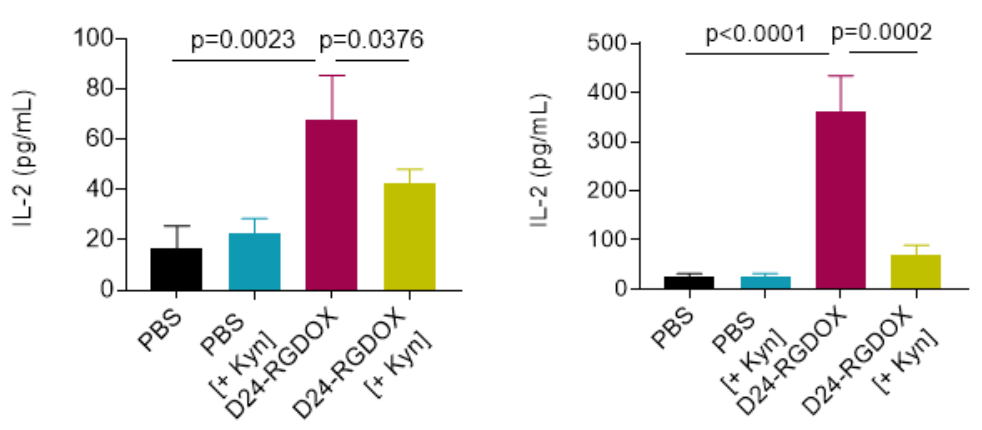
892 Fig. 8. Combined oncolytic virus and IDO inhibitor treatment enhances antiviral and

893 antitumor T cell activation. (A) Treatment timeline for the functional splenocyte assays.

894 C57BL/6 mice were intracranially (ic) implanted with GL261-5 cells and randomly assigned to

895 receive PBS, the IDO inhibitor (IDOi) indoximod, Delta-24-RGDOX, or Delta-24-RGDOX plus

896 indoximod. On day 24, splenocytes from the tumor-bearing mice were cocultured with the

897 indicated prefixed target cells for $48 \mathrm{~h}$, and the concentrations of secreted IFN $\gamma$ or IL-2 were

898 assessed by ELISA. it, intratumoral; OG, oral gavage. (B-D) Column graphs show the levels of

899 secreted IL-2 (top) or IFN $\gamma$ (bottom) in cocultures containing splenocytes from mice in the

900 indicated treatment groups and mock-infected GL261-5 cells (B), GL261-5 cells infected with

901 Delta-24-RGDOX (C), or uninfected or Delta-24-RGDOX-infected GL261-5 cells with or

902 without kynurenine (Kyn, $25 \mu \mathrm{M}$ ) supplementation in the medium (D). Data are shown as the

903 means \pm SDs $(n=3)$. Indicated P-values were evaluated using a two-tailed Student's t-test.

904 
Figure 9: Schematic illustration of the reshaping of the tumor microenvironment by the inhibition of virotherapy and IDO inhibition.

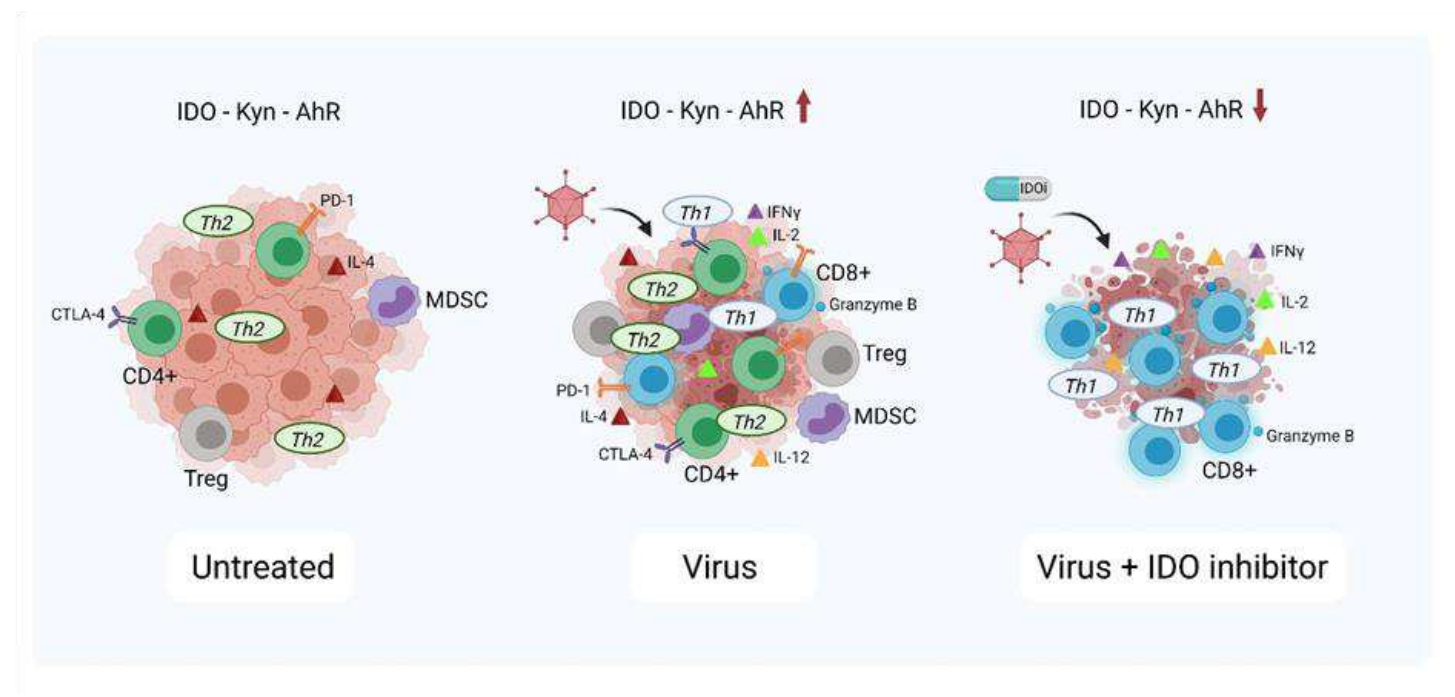

Fig. 9. Schematic illustration of the reshaping of the tumor microenvironment by the inhibitory effects of virotherapy and IDO inhibition. The glioma microenvironment is characterized by a paucity of T cells, with the few cells present skewed toward the Th2 phenotype. This Gata-3-driven Th2 phenotype promotes IL-4-based functions and the recruitment of MDSCs, Tregs, and anergic PD $-1^{+}$or CTLA $-4^{+}$T cells. The infection of gliomas with oncolytic viruses, such as Delta-24-RGDOX, induces a Th1 phenotype with increased production of IL-12 and/or IFN $\gamma$. However, viral infection activates both the IFN $\gamma$-driven IDOKyn-AhR cascade and the maintenance of immunosuppression via the activation of MDSCs and Tregs. The addition of IDO inhibitors to virotherapy results in an imbalance in signaling with a predominance of $\mathrm{Th} 1$ and $\mathrm{CD}^{+}$cytotoxic $\mathrm{T}$ cells, leading to tumor eradication. (Figure created with BioRender.com.) 


\section{Supplementary Files}

This is a list of supplementary files associated with this preprint. Click to download.

- SupplementaryDataFile1.xlsx

- SupplementaryDataFile2.xIsx

- NguyenetalSupplementaryMaterial.pdf 